INRA Prod. Anim., 2017, 30 (2), 139-152

\section{Production laitière des vaches allaitantes : caractérisation et étude des principaux facteurs de variation}

B. SEPCHAT', P. D'HOUR', J. AGABRIEL ${ }^{2}$

${ }^{\prime}$ Herbipôle, INRA, 63122, Saint-Genès-Champanelle, France ${ }^{2}$ UMR1213 Herbivores, INRA, Vetagro Sup, 63122, Saint-Genès-Champanelle, France

Courriel : bernard.sepchat@inra.fr

La production laitière des vaches allaitantes est souvent considérée par les éleveurs comme un trait génétique subi et non estimable. Le regroupement des mesures de lait bu par les veaux, réalisées depuis 15 ans dans les troupeaux charolais, limousins et salers de l'unité expérimentale Herbipôle a permis de décrire finement la lactation des mères, son évolution et ses facteurs de variations. Cette analyse souligne l'importance d'estimer ce phénotype sur un ensemble plus large de vaches.

En France, l'élevage de bovins allaitants produit majoritairement des veaux élevés sous la mère, appelés communément broutards et destinés à l'engraissement. Ceux-ci se nourrissent surtout de lait maternel et d'herbe jusqu'au sevrage vers l'âge de 7 à 10 mois. Ils sont commercialisés à l'âge de 9-12 mois pour un poids vif moyen de $320 \mathrm{~kg}$. La productivité des élevages allaitants dépend étroitement du nombre de veaux sevrés par vache et de leur poids car ils sont vendus sur ce dernier critère. La recherche de la meilleure efficience économique de ces systèmes a souvent été confondue avec la maximisation des prix de vente de ces jeunes broutards mâles dont le poids et la conformation sont bien valorisés sur le marché d'export notamment en Italie. L'alourdissement des broutards a été considéré comme un objectif de production majeur dans les élevages qui en ont fait leur spécialité, dans le charolais notamment. Ainsi le prix de vente à la tête a été souvent privilégié par rapport au prix de revient de l'animal par une recherche de croissances élevées sous la mère quelles que soient les années, et les conditions d'abondance de l'herbe. La pratique de complémentation des veaux avec de l'aliment concentré est devenue majoritaire et peut désormais débuter très tôt, même depuis l'âge de 3 mois. C'est une manière privilégiée de sécuriser la production pondérale. $\mathrm{La}$ complémentation se réalise le plus souvent avec des aliments donnés en libre-service aussi bien à l'étable qu'au pâturage en fin d'été. Cette tendance s'est observée au sein de toutes les races (Veysset et al
2005). Pourtant la croissance du veau dépend de tous les aliments dont il dispose : le lait bu, l'herbe pâturée (ou tout autre fourrage à base d'herbe) et enfin l'aliment concentré.

La période où le jeune veau est nourri sous sa mère peut être comprise entre 2 à 10 mois selon le système d'élevage et selon la race considérée. Cependant, au cours de ses trois premiers mois de vie, son alimentation est toujours essentiellement lactée. Les gains de poids réalisés par le jeune durant ses premières semaines d'élevage sont particulièrement importants puisqu'ils conditionneront la croissance future de l'animal. Un retard de croissance au cours de cette phase d'allaitement s'avère difficilement rattrapable dans la suite de la carrière de l'animal. La croissance du veau élevé au pis dépend étroitement de la quantité de lait bu au cours des tétées. La Production Laitière (PL) de la mère et la capacité de buvée du jeune veau conditionnent tous deux la croissance réalisée, car les deux interagissent fortement. Le potentiel laitier des vaches allaitantes dépend de leur niveau génétique, optimisé par des pratiques adaptées. Néanmoins, les quantités de lait produites sont régulées par le jeune à travers la fréquence et l'intensité des prélèvements effectués au cours de la tétée. C'est lui qui stimule la production ou, au contraire, la régule. Il est toujours difficile de déterminer qualitativement et quantitativement les influences respectives de la vache sur la croissance de son veau et du jeune veau sur le niveau de PL de sa mère. Un $\mathrm{kg}$ de lait bu supplémentaire correspond en moyenne pour un veau à un gain marginal de croissance journalière d'environ 60 à $90 \mathrm{~g}$, soit l'équivalent d'un apport de 250 à $300 \mathrm{~g}$ d'aliment concentré à base de céréales (Petit 1988). Au sevrage, ce gain de poids marginal réalisé grâce au lait supplémentaire n'a pas tout à fait la même composition que celui réalisé avec un aliment concentré comme l'ont montré Garcia-Launay et al (2008). Il permettrait d'accroître davantage la masse maigre (format) et sur les mâles cet effet a des conséquences intéressantes par la suite, lors de la finition (Sepchat et al 2011).

Contrairement aux vaches laitières, l'évaluation de la PL est lourde à mettre en œuvre chez les vaches allaitantes. Dans les élevages, elle peut être estimée très indirectement par le poids des jeunes veaux à âge fixe. En situation de contrôle de performances c'est le Poids à Age Type (PAT) des veaux à 120 jours qui renseigne le plus l'index laitier de la mère (France Génétique Élevage 2011). Mais $50 \%$ de la variabilité de la croissance du veau dépend aussi de son propre génotype (Ricordeau 1992). En station expérimentale, la PL des vaches allaitantes peut être estimée à partir la mesure du lait bu par le ou les jeune(s) obtenue par pesée avant et après tétée lors de séances d'allaitements surveillés. Les conditions rigoureuses de cette méthode ont été détaillées par Le Neindre et Dubroeucq (1973) et n'ont pas évolué depuis. Toutefois, elle reste coûteuse en temps et en main-d'œuvre et n'est pas 
généralisable aux élevages commerciaux.

Les objectifs de cet article sont de décrire les courbes de lactation, de caractériser leurs facteurs de variation et $d$ 'analyser les relations entre les lactations des vaches et la croissance de leurs veaux dans les trois races françaises dont nous disposions à l'unité expérimentale Herbipôle de l'Inra. Après avoir rappelé les principes et les conditions pour estimer la quantité produite, nous ferons le point des connaissances spécifiques sur la lactation des vaches qui allaitent, avant d'en tirer au final des recommandations pratiques destinées à conforter les innovations qui permettront d'élargir la base des animaux phénotypés sur ce caractère.

\section{1 / Les données disponibles et leur traitement}

\section{1 / La mesure de la production de lait chez les vaches allaitantes}

La PL peut être estimée soit par la traite, soit par la pesée du veau avant et après une tétée surveillée.

La traite manuelle ou mécanique pose un problème d'acceptation par les vaches. Lors de la traite, le veau peut éventuellement stimuler le démarrage de la production, comme pour la traite des vaches salers, mais la mesure du lait trait correspond alors à ce que la vache veut bien donner et non à ce qu'elle donne réellement chaque jour à boire à son veau. Ce problème a conduit à la mise au point de la seconde méthode par double pesée du veau.

\section{a) La traite}

Réalisée en présence ou en absence du veau, la traite manuelle est la technique la plus ancienne pour évaluer la PL des vaches allaitantes. Elle a ensuite été mécanisée par exemple dans les travaux de Gleddie et Berg (1968) et peut être précédée ou suivie rapidement d'une injection d'ocytocine si on vise à vider complètement la mamelle. Sans injection hormonale, Somerville et Lowman (1980) considèrent les résultats comme peu précis. Avec injection d'ocytocine Rodrigues et al (2014) montrent que cette méthode ne permet pas d'obtenir la forme des courbes de lactation d'une vache qui allaite, comme celle obtenue avec le contrôle par pesées du veau. Enfin, la traite reste une technique qui nécessite de manipuler les vaches. Elle est donc difficilement réalisable avec les troupeaux de grande taille (Rodrigues et al 2014). Son utilisation pour un phénotypage à grande échelle de la PL chez les vaches allaitantes n'est donc pas possible.

\section{b) Le contrôle laitier par « double pesée »}

Le Contrôle Laitier (CL) par « double pesée » avant et après la tétée est retenu comme méthode de référence par Le Neindre (1974) et appliquée depuis cette date dans les installations expérimentales de l'Inra. C'est celle utilisée dans toutes les données analysées dans notre étude (cf. encadré). La méthode donne une estimation correcte du lait bu et donc produit, même si elle n'estime pas obligatoirement les pertes éventuelles (urines, fèces) survenues entre les pesées. Les valeurs obtenues sont souvent supérieures à celles estimées par la traite (Le Neindre et Petit 1975b, Somerville et Lowman 1980, Mondragon et al 1983) alors que les résultats indiquant l'inverse (Belcher et al 1980) sont peu fréquents.

La fiabilité de la méthode diminue avec le développement du veau du fait des erreurs relatives de pesées. Les différences de poids mesurées avant et après tétées sont en effet d'autant plus faibles que le veau est lui-même plus lourd. Pour éviter cela le nombre de tétées surveillées est réduite à un, au-delà de $300 \mathrm{~kg}$ pour que la quantité de lait bu soit suffisante. Dans le très jeune âge, par contre c'est l'effet de la contrainte de la limitation à deux tétées par jour qui pose questions. Si les tétées surveillées étaient réalisées à intervalle fixe toutes les cinq heures, elles seraient plus proches du rythme naturel de tétée d'un jeune veau (4 tétées par 24 heures). Toutefois cette pratique fastidieuse à réaliser par la multiplication des pesées, est aussi jugée plus imprécise en raison de la perturbation des comportements des veaux qu'elle nécessite (Le Neindre et Petit 1975b).

Enfin le jour du CL, il est possible que la sécrétion lactée soit toujours modifiée par la contrainte forcée des deux tétées : les veaux ne pourraient pas boire tout le lait stocké dans la mamelle, surtout si leurs mères sont fortes productrices ou, à l'opposé, les mères seraient stimulées par la séparation forcée avant la mesure, et la mesure surestimerait le lait réellement bu. Ainsi nous avons constaté que des contrôles de production réalisés 3 jours consécutifs (J1, J2, J3) sur des veaux habitués à rester en permanence avec leurs mères, ont des valeurs systématiquement supérieures le premier jour par rapport aux jours suivants, respectivement 7,14 (J1) vs $6,65(\mathrm{~J} 2)$ et $6,67 \mathrm{~kg} / \mathrm{j}$ (J3) $(\mathrm{SEM}=0,31, n=16$ veaux $)$. Cet écart $(\mathrm{J} 1, \mathrm{~J} 2)$ de $0,5 \mathrm{~kg} / \mathrm{j}$ suggère que le CL perturbe le comportement du couple mère veau lors de la tétée, sans toutefois qu'on en ait l'assurance expérimentale, car cette observation a toujours été faite dans le même environnement et la même

\section{Encadré. Principe de la mesure indirecte de la production laitière journalière} $(P L)$ par 2 tétées surveillées en 24 h (d'après Le Neindre 1973).

Le Contrôle Laitier (CL) est réalisé sur un intervalle d'environ 24 heures. II commence par la séparation du veau de sa mère après les tétées le jour $0\left(\mathrm{~J}_{0}\right)$ l'heure $H\left(H_{0}\right)$ de la séparation, le soir de $J_{0}$ est notée. Le lendemain jour $J_{1}$, un opérateur réalise deux tétées surveillées le matin et le soir (à l'heure $\mathrm{H}_{1}$ ). Chacune est encadrée par une pesée avant et après la buvée dont on calcule les différences $\mathrm{d} 1$ et $\mathrm{d} 2$. Entre ces deux tétées le veau est parqué, sans possibilité d'approcher sa mère. La somme des différences $(\mathrm{d} 1+\mathrm{d} 2)$ de poids du veau est ramenée à 24 heures en considérant l'heure de démarrage de la surveillance $\mathrm{H}_{0}$ à $\mathrm{J}_{0}$.

$$
P L(k g / j)=\frac{(d 1+d 2) \times 24}{H_{1}-H_{0}}
$$

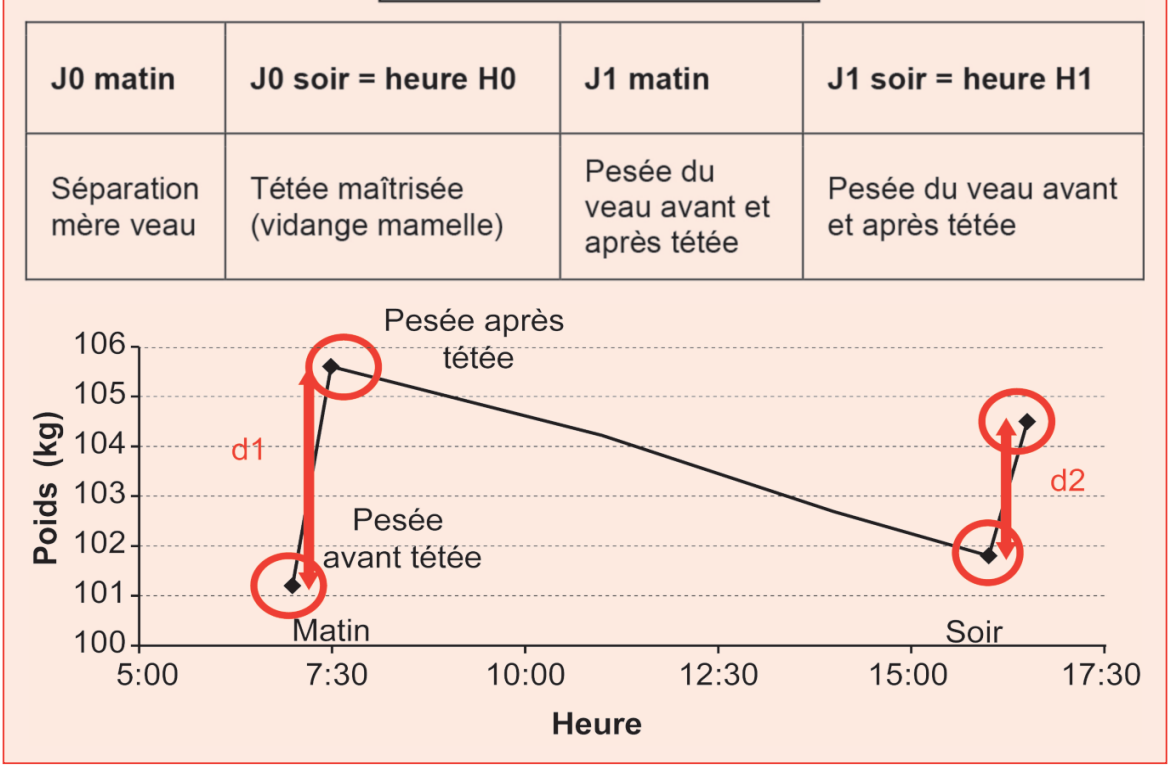


étable. Elle interroge néanmoins sur la valeur absolue du contrôle lorsqu'il est fait une seule fois en 24 heures sur des vaches habituellement en stabulation libre, mode d'hébergement désormais le plus classique. Mais Le Neindre (1974) considère que le veau de plus de deux mois (100 kg et plus) est toujours capable de boire en deux tétées tout le lait produit par sa mère en 24 heures. Ainsi, même si on note un certain manque de précision de la méthode, celle-ci apparaît comme la plus simple à mettre en place de façon régulière et sur de grands effectifs.

\section{2 / Production de lait bu et courbe de lactation}

Les données disponibles de mesure du lait produit par des vaches charolaises, salers et limousines sont toutes issues des sites expérimentaux de l'UE Herbipôle. Elles ont été réalisées depuis l'année 1990 et sont regroupées dans la base de données zootechniques «Aladin » (Application de gestion des troupeaux Laitiers et Allaitants des Domaines Inra). Cette base de données conçue sous Microsoft ACCESS rassemble les informations individuelles de gestion des animaux de leur naissance à leur sortie (mise bas et ses conditions, nombre de veaux par vêlage, date de mise à l'herbe, localisation...), ainsi que les mesures individuelles (pesées, Notes d'État Corporel (NEC) et éventuellement alimentation). Par convention, les vêlages entre le 1 er décembre et le 14 janvier sont définis comme des vêlages précoces alors que les vêlages du 15 janvier au $1^{\text {er }}$ mars correspondent à des vêlages d'hiver « classiques ». La base de données contient plus de 5000 lactations, mais seules 1292 ont été retenues pour l'analyse. Les lactations avec moins de 7 contrôles laitiers par vache non correctement répartis sur la durée de la lactation ont été supprimées afin d'avoir une estimation

Figure 1. Caractéristiques de la courbe de lactation d'une vache allaitante vêlant en hiver.

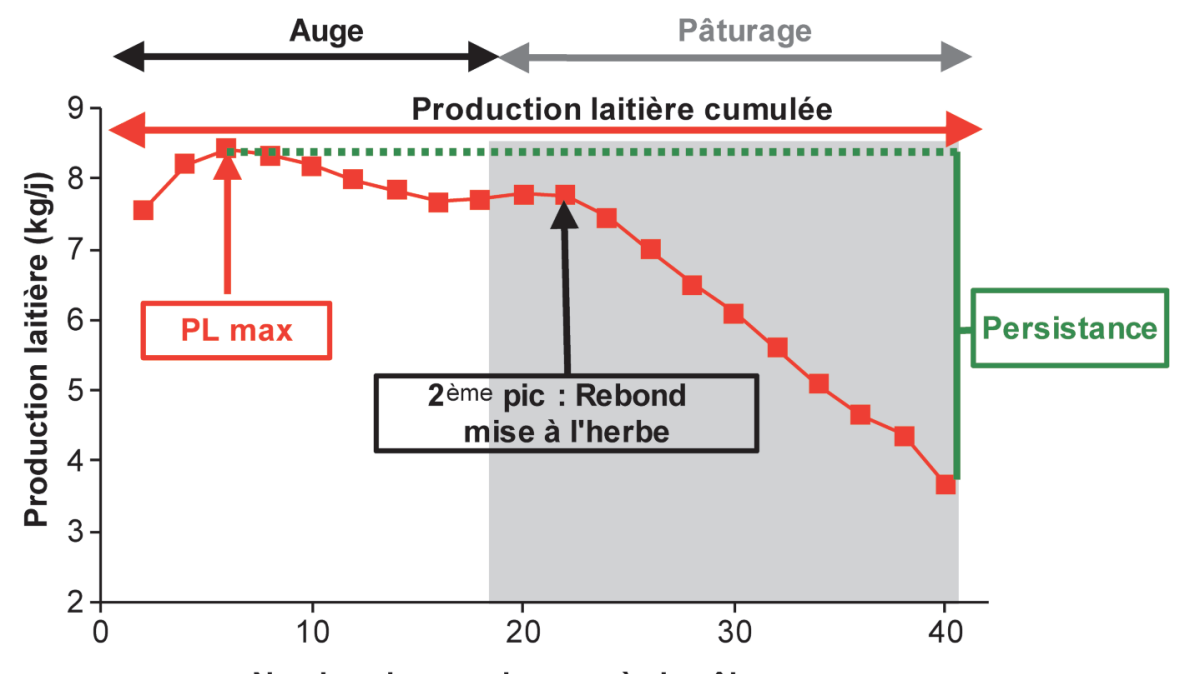

Nombre de semaines après le vêlage correcte de la PL totale et de la production cumulée aux périodes types $90 \mathrm{j}$, $120 \mathrm{j}$ et $210 \mathrm{j}$. Les lactations avec moins de 4 contrôles après la mise à l'herbe ont également été supprimées. Mais grâce à un sous-ensemble de 28 vaches contrôlées une fois par semaine au pâturage, nous avons pu noter que la baisse de PL est relativement linéaire après la mise à l'herbe, au moins lorsque celleci est effectuée après le troisième mois de lactation. Cette observation nous a permis de conserver les lactations de vaches dont un seul contrôle au pâturage manquait. La production intermédiaire manquante a été estimée par extrapolation linéaire entre deux mesures réalisées à deux mois d'intervalle tout au plus.

\section{3 / Caractéristiques de la lac- tation : maximum, persistance production totale}

Les caractéristiques de la PL mesurées ont été analysées en considérant les variables positionnées dans la figure 1 : production maximale (PL max), totale (PLT), PERSistance (PERS) et Rebond à la Mise à l'Herbe (RMH).

Contrairement à celle des vaches laitières, la PL des vaches allaitantes augmente lentement après le vêlage pour atteindre un maximum situé entre le premier et le troisième mois de lactation. Celui-ci dépend de l'équilibre existant entre la capacité du jeune veau à boire le lait et le potentiel de production de l'animal (Blanc et al 1999). La PL maximale a donc été déterminée pour chaque lactation, en considérant sa semaine d'occurrence. La recherche d'un ou plusieurs maximums du fait d'un rebond observé après la mise à l'herbe a d'abord été réalisée en considérant la période 90120 jours (la plus fréquente) puis en l'élargissant à la période 49-140 jours.
L'analyse détaillée de la persistance permet de mieux quantifier la fin de lactation. Elle peut se définir comme le rapport entre la PL d'un mois et celle du mois précédent. Celui-ci varie de 94 à $96 \%$ chez la vache laitière (Boujenane 2010) et a été estimée entre 90 et $95 \%$ chez la vache allaitante (Le Neindre et al 1976). Nous avons calculé une variable (négative) PERS de décroissance de la production par jour, à partir de la formule de Jenkins et al (2000) : considérant PY $=$ PLmax et FY la valeur minimum de production en fin de de lactation :

$$
\begin{gathered}
\text { PERS }=(F Y-P Y) \times 1000 / D(g / j), \\
\text { avec PERS négatif }
\end{gathered}
$$

On a $F Y=P L$ minimale en fin de lactation $(\mathrm{kg} / \mathrm{j}), \mathrm{PY}=\mathrm{PL}$ maximale au pic de lactation $(\mathrm{kg} / \mathrm{j})$, et $\mathrm{D}$ est l'écart en jours entre PY et FY. D = (T2-T1), avec T2 la date calendaire moyenne des 3 derniers contrôles et $\mathrm{T} 1$ la date calendaire moyenne des trois contrôles entourant le pic de lactation.

Cette formule a été adaptée à nos données en calculant la production de lait maximale $P Y$ à partir du deuxième maximum, s'il existait, et en retenant la moyenne des trois contrôles entourant le pic considéré. La valeur moyenne FY a été prise comme moyenne des trois derniers contrôles avant sevrage afin de limiter les erreurs dues à un seul mauvais contrôle de fin de lactation.

\section{4 / Relation entre la croissance du veau et la lactation de la mère}

Afin de mettre en relation la croissance des veaux et la PL des mères, une liste de 1578 veaux salers et limousins (nés avant le $01 / 10 / 2013$ ) et de 1553 veaux charolais (nés avant le 01/06/2013) a été extraite de la base de données Aladin. De cette liste ont été retirés les veaux jumeaux ou triplets, qui ne servent pas pour l'étude. Un fichier « vache-veau » a été constitué qui regroupe, d'une part, les caractéristiques de la mise bas, la date et la période de vêlage, le numéro de lactation de la vache et, d'autre part, les mesures sur le veau, sexe, poids à la naissance, poids à âges types (PAT à 90 , 120 et 210 jours) ainsi que les Gains Moyens Quotidiens (GMQ) calculés entre ces âges types. Pour les veaux dont le poids n'a pas été mesuré lors d'une semaine correspondant à un PAT, nous avons réalisé une régression linéaire à partir des 5 pesées entourant ce PAT. Les veaux n'ayant pas fait l'objet de pesées assez proches de la semaine type pour faire une estimation correcte ont alors été retirés de l'étude. En parallèle, les PL des mères correspondant aux dates de ces PAT $(90,120$ et 210 j) ont été calculées afin d'être associées aux GMQ. 
Pour cela, on a considéré le cumul des productions, soit la PL journalière moyenne entre deux contrôles que l'on multiplie par le nombre de jours de l'intervalle. Finalement, 281 couples mère/ veau salers, 182 couples mère/veau limousins et 410 couples mère/veau charolais ont été utilisés pour l'analyse statistique de la relation mère-veau.

\section{5 / Niveau d'alimentation de la vache et lactation}

Pour établir le lien entre PL et niveau d'alimentation de la mère, nous avons regroupé les données individuelles de cinq expérimentations récentes ${ }^{1}$ où 18 lots de vaches charolaises (soit 115 vaches) recevaient individuellement après vêlage et à l'étable des niveaux alimentaires très contrastés (de 3 à 5 UFL d'écart). La production de lait était mesurée au moins 1 fois par semaine par la méthode des tétées surveillées. Les durées d'application moyenne des traitements alimentaires étaient de 117 jours (minimum 101, maximum 130) à partir du septième jour de lactation.

\section{6 / Ajustements à un modèle}

Le modèle de lactation retenu est celui proposé par Coulon et Pérochon 2000 dans lequel trois effets majeurs sont considérés : la parité, le potentiel au pic, la date de mise bas. La forme mathématique de ce modèle considère une production maximale (PLmax) équivalente à PY soumise à des effets liés au stade de lactation (effet exponentiel décroissant) et des effets liés à la saison (durée du jour : effet sinusoïdal). Elle avait été développée pour des vaches laitières afin de s'ajuster à des formes de courbes de production individuelles très variées en conditions expérimentales changeantes. Mais ici les paramètres n'ont pas été ajustés à chaque individu car pour une même vache, d'un contrôle à l'autre, il y a de fortes variabilités dans les productions qui sont davantage liées à la méthode de mesure qu'à la lactation elle-même. Les courbes de lactations individuelles n'auraient pas de sens. Nous avons préféré ajuster globalement les courbes de productions journalières moyennes pour les 3 races disponibles dans nos données et selon la parité. Ce modèle a été ajusté sur un double critère, d'une part, en minimisant la somme des carrés des écarts (observé-prédit) par pas de 15 jours, et d'autre part, en comparant les valeurs estimées observées/prédites de la PL Totale. Les résultats fournissent une référence de l'évolution de la production par race et parité (primipares $v s$ multipares) qui a ensuite servi à l'élabo- ration des recommandations alimentaires Inra 2017 (Agabriel et De la Torre 2017).

\section{2 / La courbe de lactation et ses facteurs de variations}

\section{1 / Le niveau de production et son évolution dans le temps}

La courbe de lactation d'une vache allaitante (figure 2) est plus plate qu'une courbe classique de vache laitière et elle présente souvent un deuxième maximum après la mise à l'herbe. Les facteurs modulant la forme de la courbe de lactation et les niveaux de production sont abordés successivement.

\section{a) Effet de la race}

La quantité de lait produit varie surtout avec le potentiel génétique de l'animal (Petit et al 1994). Dans nos données les PLT moyennes des trois races Salers, Limousine, Charolaise sont significativement différentes. Pour des durées respectives de $271 \pm 21,276 \pm 19$ et 266 \pm 26 jours de lactation, elles s'élèvent à $2247 \pm 470 \mathrm{~kg}, 1628 \pm 313 \mathrm{~kg}$, et 1840 $\pm 355 \mathrm{~kg}$, soit $8,3 \pm 2,1 ; 5,9 \pm 1,2$ et 6,9 $\pm 1,3 \mathrm{~kg} / \mathrm{j}$ (variabilité calculée entre individus). Les vaches charolaises et limousines qui sont les races les plus typées pour la production de viande ont une production inférieure aux vaches salers. Les PLmax des trois races sont respectivement de 10,4 (Salers), 8,3 (Limousine) et $10,3 \mathrm{~kg} / \mathrm{j}$ (Charolaise) pour les multipares, et de 9,$5 ; 7,4$ et $8,9 \mathrm{~kg} / \mathrm{j}$ pour les primipares. La variabilité individuelle des PL se situe autour de $20 \%$. Elle indique qu'une sélection efficace sur cette performance serait possible à condition de disposer de cette information.
Ces résultats sont à comparer à ceux de l'ouvrage des recommandations alimentaires aux USA (NRC 2016) qui indique pour ces mêmes trois races, une même valeur maximale $9 \mathrm{~kg} / \mathrm{j}$, légèrement supérieure aux valeurs données pour les races britanniques Angus $(8 \mathrm{~kg} / \mathrm{j})$ ou Hereford $(7 \mathrm{~kg} / \mathrm{j})$. Mais ces informations ne reposent pour nos races que sur une seule publication (Jenkins et Ferrell 1992) dans laquelle 16 individus par race avaient été mesurés. En Irlande, Mc Gee (1997) a regroupé sept comparaisons publiées entre vaches de races charolaises et angus. Dans trois comparaisons la charolaise produisait environ $1 \mathrm{~kg} / \mathrm{j}$ de plus que la vache de race angus, dans deux comparaisons $0,9 \mathrm{~kg} / \mathrm{j}$ de moins, les productions étant égales dans les deux autres. Selon ces données, les productions des deux races seraient alors égales à $7,1 \mathrm{~kg} / \mathrm{j}$ (moyenne des données citées), supérieures à celles de la race Hereford (6,3 kg/j, 9 publications citées). La comparaison avec les autres races françaises est plus difficile par manque de données (Blonde d'Aquitaine) ou du fait de l'ancienneté des données qui ne correspondent plus avec les génotypes actuels (formats très inférieurs). Ainsi la comparaison citée réalisée dans les années 1970 à l'Inra de Bourges (Petit et Liénard 1988) indiquait en moyenne de lactation, un niveau de production de la race Maine Anjou de $6,7 \mathrm{~kg} / \mathrm{j}$, de $6 \mathrm{~kg} / \mathrm{j}$ pour les races charolaise et aubrac et de $5,1 \mathrm{~kg} / \mathrm{j}$ pour la race limousine.

Bien évidemment le croisement entre race à viande et race laitière reste le moyen le plus efficace pour accroître significativement et rapidement la PL. Ce type de croisement est souvent réalisé en Irlande (Mc Gee et al 2005) et permet une amélioration de +20 à $+35 \%$ $(10,5$ à $12.5 \mathrm{~kg} / \mathrm{j})$ de la PL observée sur les vaches de races à viande.

Figure 2. Courbes de lactation moyenne mesurées entre 1999 et 2015 sur des vaches de race salers, limousine et charolaise à l'UE Herbipôle (parités confondues).

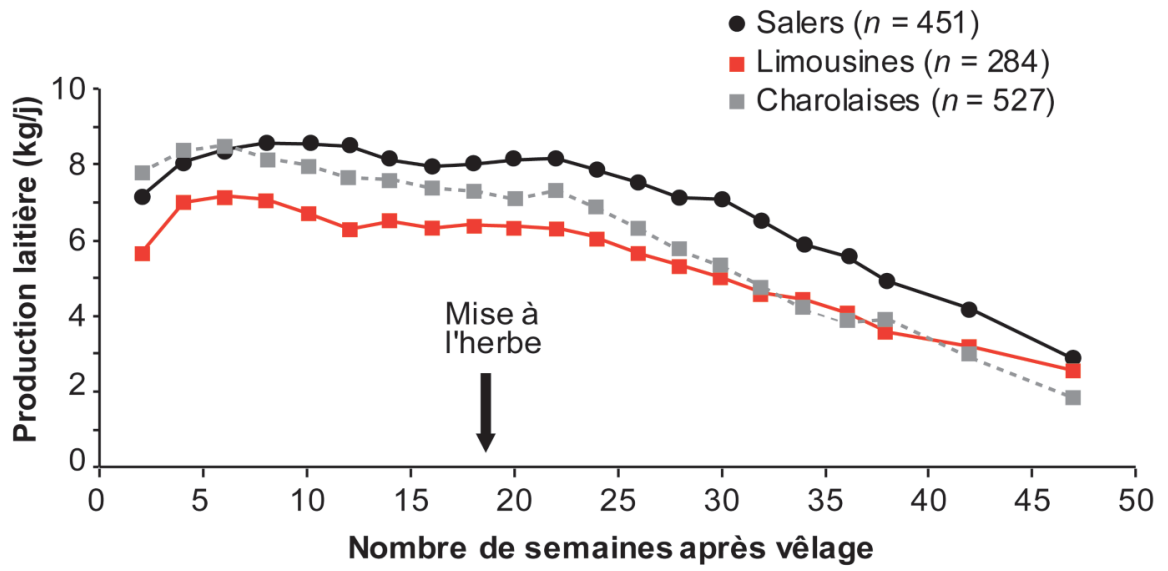

${ }^{1}$ Données mises à disposition par Anne De La Torre. 
Figure 3. Répartition des semaines auxquelles intervient le pic de lactation (PLmax) selon la race (toutes lactations confondues).

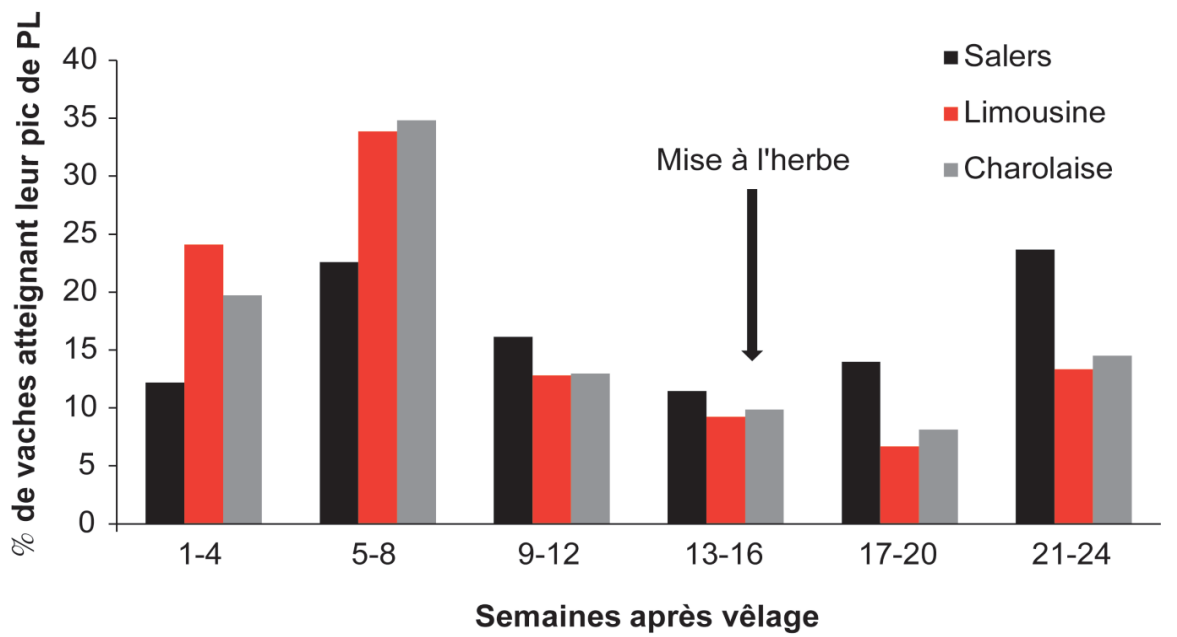

L'analyse des fréquences de l'occurrence du pic de lactation (en semaines) montre que 22 à $23 \%$ des vaches salers, tous numéros de lactation confondus, présentent leur PLmax entre les semaines 5 et 8 après la mise bas. Cela correspond à l'occurrence de la réalisation du pic de production d'une vache laitière.

Souvent la forme de la courbe est bimodale et $23,7 \%$ des vaches expriment leur PLmax après la Mise à l'Herbe (MH), entre la $21^{\text {ème }}$ et la $24^{\text {ème }}$ semaine après mise bas. Les vaches limousines et charolaises l'atteignent majoritairement $(33,9$ et $34,8 \%$ ) entre les semaines 5 et 8 et
13,3 et $14,5 \%$ entre les semaines 21 et 24 (figure 3 ).

La distribution de l'occurrence des pics de lactation est significativement différente entre la race salers et les deux autres races (test du chi 2), sans qu'il n'y ait de différence entre les vaches limousines et charolaises sur ce critère. Il y a aussi une différence entre races sur la forme et l'importance du rebond de PL après la mise à l'herbe toujours plus accentué en race salers. Ce rebond arrive en moyenne 20 jours après la mise à l'herbe (voir paragraphe 2.2).
Comparés à ceux de Le Neindre et al (1976), nos résultats montrent une diminution globale de la PL des trois races mesurées depuis 40 ans. En utilisant la même technique de tétée surveillée, et dans les mêmes installations expérimentales, Le Neindre et al (1976) mesuraient respectivement des PL de 10,5 et $6,6 \mathrm{~kg}$ pour les salers et les limousines. La diminution s'explique certainement en partie par l'accent mis sur l'amélioration génétique des performances bouchères, car dans le même temps les vaches ont en moyenne augmenté leur format de +5 à $+6 \mathrm{~kg} / \mathrm{an}$ quelle que soit la race. En effet, la PL est corrélée négativement avec le rendement carcasse : $\mathrm{r}=-0,2$ (Colleau et al 1983).

Ajusté aux 6 jeux de données ( 3 races, 2 parités) le modèle de lactation reproduit la forme d'ensemble (figure 4) mais ne permet pas de simuler le deuxième pic qui suit la mise à l'herbe. Le potentiel de production journalier ajusté est de $8,8 \mathrm{~kg}$ pour les charolaises multipares, $9,6 \mathrm{~kg}$ pour les salers et $7,8 \mathrm{~kg}$ pour les limousines (tableau 1).

\section{b) Effet de la parité et du numéro de lactation}

Quelle que soit la race, la PLT des vaches multipares est plus élevée que celle des primipares (figure 5) sans qu'il soit nécessaire de différencier les numéros

Figure 4. Ajustement des données moyennes de production de lait bu par un modèle mathématique adapté aux productions laitières de montagne (d'après Pérochon et al 1996).
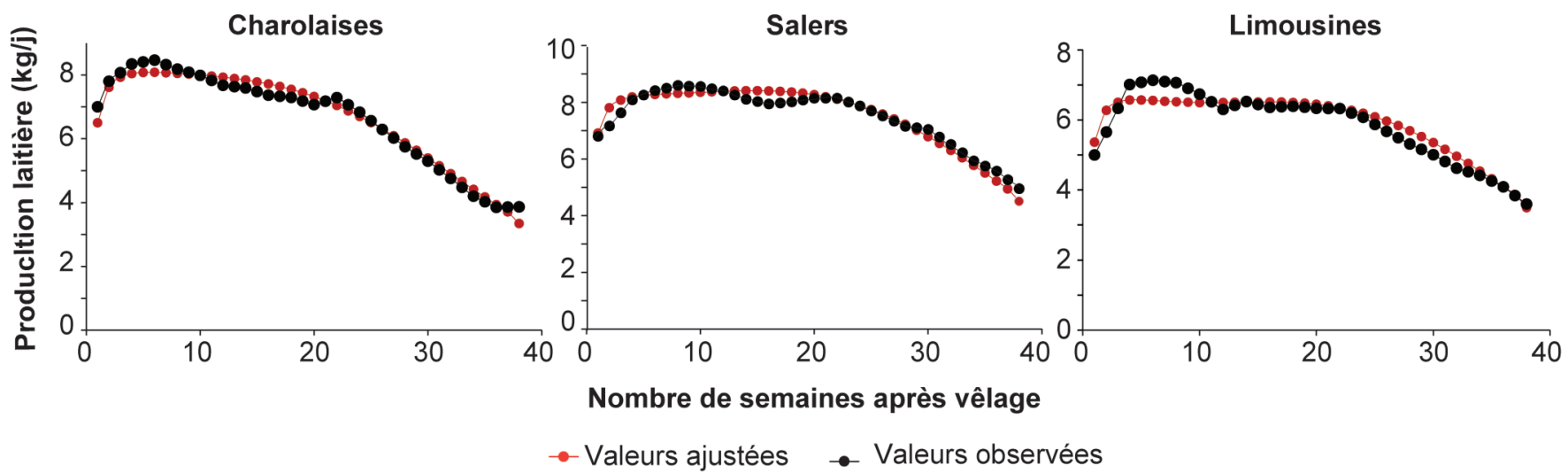

Tableau 1. Paramètres d'ajustement du modèle de lactation : production maximale et occurrence du vêlage.

\begin{tabular}{|l|c|c|c|c|c|}
\hline Groupe analysé & $\begin{array}{c}\text { Charolaises } \\
\text { Primipares }\end{array}$ & $\begin{array}{c}\text { Charolaises } \\
\text { multipares }\end{array}$ & $\begin{array}{c}\text { Charolaises } \\
\text { (toutes) }\end{array}$ & $\begin{array}{c}\text { Salers } \\
\text { (toutes) }\end{array}$ & $\begin{array}{c}\text { Limousines } \\
\text { (toutes) }\end{array}$ \\
\hline $\begin{array}{l}\text { Production maximale } \\
\text { ajustée (kg/j) }\end{array}$ & 7,9 & 9,2 & 8,8 & 9,6 & 7,8 \\
\hline $\begin{array}{l}\text { Semaine calendaire } \\
\text { de vêlage }{ }_{(*)}^{(\text {Semvel) }}\end{array}$ & 2 & 4 & 4 & 0 & 0 \\
\hline
\end{tabular}

(*) Semaine calendaire du vêlage : elle est liée à la semaine où l'insémination fécondante est considérée comme réalisée. Les vaches de race salers s'ajustent sur les paramètres publiés pour la race montbéliarde. 
Figure 5. Effet de la parité sur la courbe de lactation (lait bu, toutes races confondues).

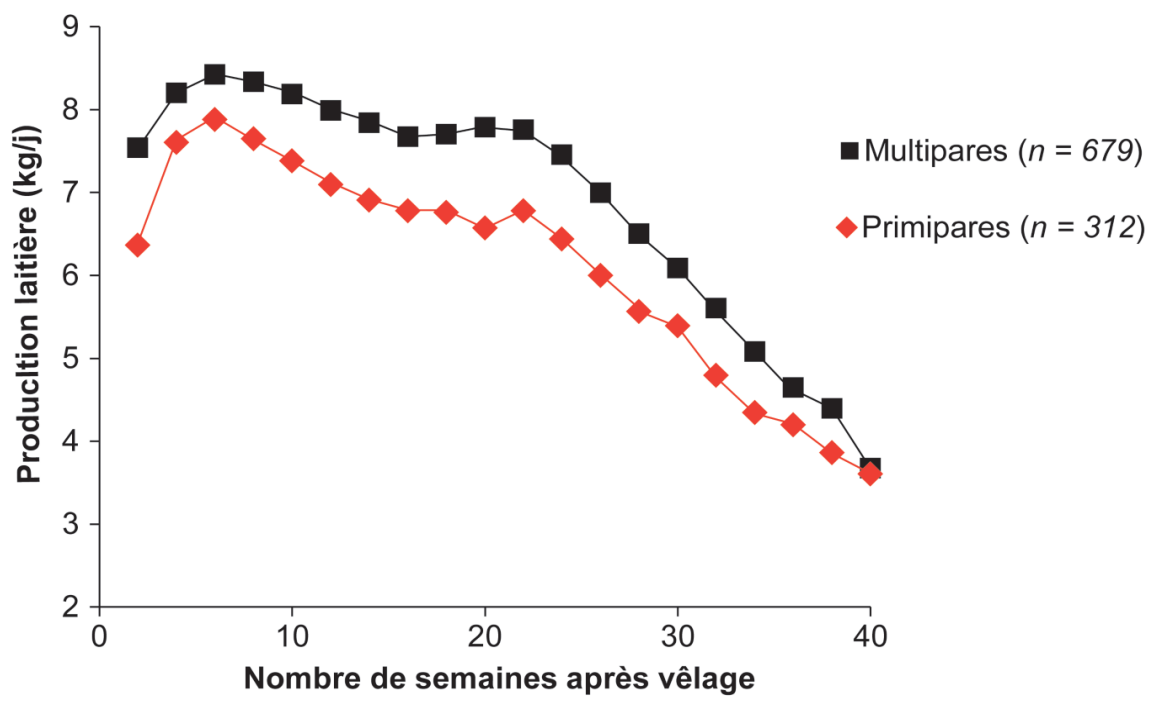

Tableau 2. Production laitière totale selon la race et la parité pour les vaches dont la durée moyenne de lactation est de $273 \pm 25 j$.

\begin{tabular}{|l|l|l|c|c|c|c|}
\hline \multirow{2}{*}{ Race } & \multirow{2}{*}{ Parité } & \multirow{2}{*}{ Nombre } & \multicolumn{2}{|c|}{$\begin{array}{c}\text { Production laitière } \\
\text { totale (kg) }\end{array}$} & \multicolumn{2}{|c|}{$\begin{array}{c}\text { Production laitière } \\
\text { moyenne (kg/j) }\end{array}$} \\
\cline { 4 - 8 } & & & Moyenne & Écart type & Moyenne & Écart type \\
\hline \multirow{3}{*}{ Salers } & Multipare & 372 & 2298 & 478 & 8,5 & 2,2 \\
\cline { 2 - 8 } & Primipare & 79 & 2008 & 343 & 7,3 & 1,4 \\
\cline { 2 - 8 } & Total & 451 & 2247 & 470 & 8,3 & 2,1 \\
\hline \multirow{4}{*}{ Limousine } & Multipare & 232 & 1661 & 310 & 6,1 & 1,2 \\
\cline { 2 - 8 } & Primipare & 52 & 1482 & 287 & 5,3 & 0,9 \\
\cline { 2 - 8 } & Total & 284 & 1628 & 313 & 5,9 & 1,2 \\
\hline \multirow{3}{*}{ Charolaise } & Multipare & 299 & 1932 & 338 & 7,3 & 1,3 \\
\cline { 2 - 8 } & Primipare & 128 & 1732 & 344 & 6,5 & 1,3 \\
\cline { 2 - 8 } & Total & 527 & 1840 & 355 & 6,9 & 1,3 \\
\hline
\end{tabular}

Figure 6. Évolution de la production laitière moyenne autour de la mise à l'herbe des vaches salers selon la parité.

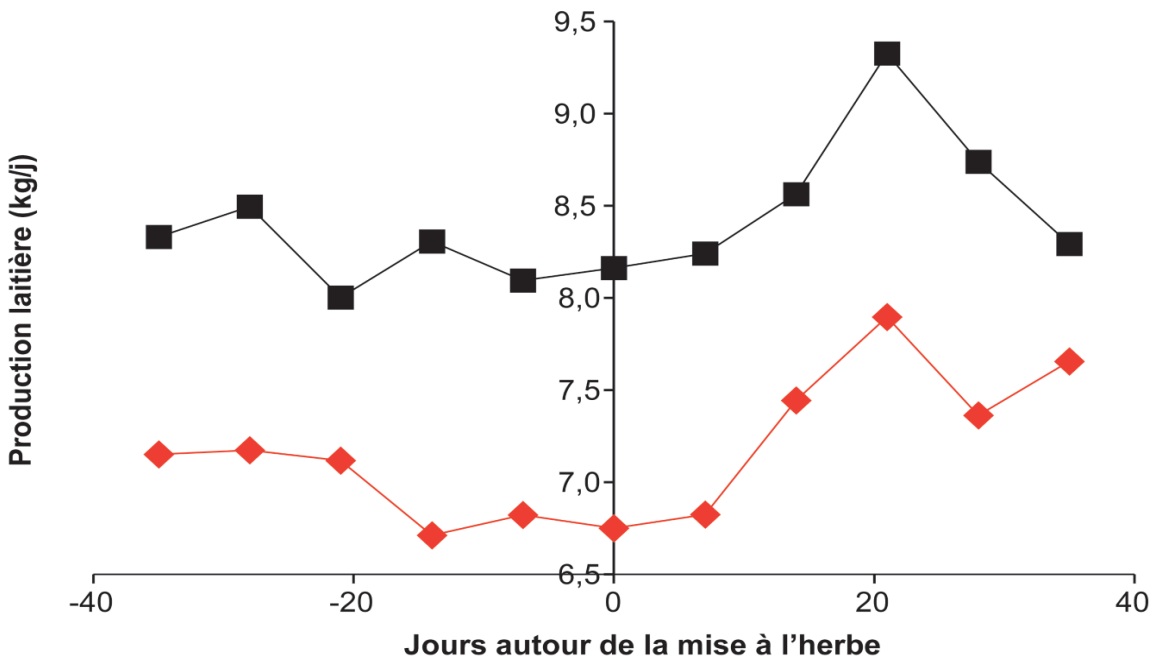

Jours autour de la mise à l'herbe de lactation des multipares (tableau 2). La PL journalière moyenne des primipares salers est ainsi de 7,3 $\pm 1,4 \mathrm{~kg} / \mathrm{j}$ et celle des multipares est de $8,5 \pm$ $2,2 \mathrm{~kg} / \mathrm{j}$.

Les primipares de race salers, limousines et charolaises produisent ainsi respectivement 280,179 et $200 \mathrm{~kg}$ de moins que les multipares soit entre 11 et $12 \%$ de moins, les écarts entre races se maintenant chez les multipares. La raison généralement évoquée de cette différence repose sur le développement de la glande mammaire qui serait métaboliquement plus active chez les multipares surtout au début de lactation et jusqu'au $1^{\text {er }}$ pic. Ceci suggère aussi qu'en début de lactation, on pourrait avoir une plus faible densité de cellules sécrétrices dans le parenchyme mammaire (Miller et al 2006).

Dans les données plus anciennes, on note que la PL maximale des vaches salers est atteinte à la quatrième lactation à 6 et 7 ans d'âge (Petit et al 1984) et diminue au-delà. Le Neindre et al (1976) notent également des hausses de production de $+28 \%$ entre les lactations 1 et $4,+11 \%$ entre les lactations 1 et 2 tandis que la PL diminue au-delà de la septième lactation. Cette hausse de la PL après la première lactation serait cependant moins marquée chez les races rustiques que chez les races à viande (Le Neindre 1974, Ménissier et al 1992). Ces différences tranchées au-delà de la deuxième lactation ne se sont pas retrouvées dans notre jeu d'observations où le numéro de lactation n'affectait pas significativement la PL.

\section{c) Effet de la période de vêlage}

À même numéro de lactation, pour des vêlages d'hiver, les vaches salers ayant mis bas précocement ont une PL journalière moyenne inférieure à celle des vaches ayant vêlé à une date classique $(7,0 \pm 1,5 \mathrm{~kg} / \mathrm{j}$ vs $7,7 \pm 1,7 \mathrm{~kg} / \mathrm{j}, \mathrm{p}<$ $0,05)$. Ce n'est qu'une tendance pour les vaches limousines $(5,5 \pm 1 \mathrm{~kg}$ vs $6,1 \pm$ $1,3 \mathrm{~kg}$ ) qui se retrouve peu sur les charolaises $(6,4 \pm 1,5 \mathrm{~kg}$ vs $6,7 \pm 1,4 \mathrm{~kg})$. Il convient de remarquer que, quelle que soit la race, la durée de lactation des vaches vêlant précocement $(292 \pm 18 \mathrm{j})$ est globalement supérieure à celle des vaches vêlant à une date classique (264 $\pm 18 \mathrm{j}$ ). Ceci résulte de la conduite des troupeaux, les vaches étant en effet toutes taries simultanément au moment de la rentrée à l'étable. L'effet de cet écart de durée de lactation est néanmoins à minimiser puisqu'il ne joue que sur $1,5 \mathrm{~kg}$ de lait en plus par jour de lactation supplémentaire soit $42 \mathrm{~kg}$ sur le total de la lactation (vaches salers). 


\section{2 / Le rebond de production à la mise à l'herbe}

Durant le pâturage de printemps, la production de la vache allaitante est capable de rebondir pour atteindre un second pic de lactation, souvent plus élevé que le premier. Si le premier pic est atteint aux alentours du 45 ème jour de lactation, le rebond de production arrive en moyenne 20 jours après la mise à l'herbe, aux alentours du 130 ème jour de lactation. Il est d'intensité variable selon les races soit respectivement $+1,2$ $+0,9 \mathrm{~kg} / \mathrm{j}$ pour les salers, charolaises et nul pour les limousines. Il est également supérieur chez les multipares que chez les primipares (en moyenne $+0,7$ $\mathrm{kg}$ pour multipares de race salers par rapport aux primipares, figure 6). Plus la mise à l'herbe est éloignée du vêlage moins la réponse est marquée (effet date de vêlage sur l'intensité du $2^{\text {ème }}$ rebond $\mathrm{p}<0,01)$.

Ce second pic serait lié à la modification du statut nutritionnel des vaches avec à la fois un niveau d'ingestion accru et une valeur énergétique de la MS ingérée supérieure à l'herbe en interaction avec le stade de lactation. Comme Le Neindre et al (1976) ont pu l'observer, le printemps est une période favorable puisque l'herbe très ingestible lorsqu'elle est pâturée au bon stade (1 UEB, 1 UFL et $100 \mathrm{~g}$ de PDIE/kg MS) permet de couvrir largement les besoins énergétiques et azotés (pour près de 15 à $20 \mathrm{~kg}$ de lait). Plus la date de vêlage est proche de la mise à l'herbe plus la mamelle serait réactive, ainsi les vaches au potentiel laitier plus élevé auraient un rebond de production plus accentué (D'Hour et al 1995). La vache allaitante est ainsi capable de rebondir dans des conditions d'alimentation plus favorables, et les jeunes veaux peuvent tirer bénéfice de cette période particulière.

\section{3 / La persistance de la lactation}

Une fois que le pic de lactation est atteint, la PL décline avec l'avancée de la lactation. Selon la formule proposée au $\S 1,3$, les persistances des limousines $(-17,7 \pm 5,9 \mathrm{~g} / \mathrm{j})$, des salers $(-21,6 \pm$

Figure 7. Relation entre la persistance de la production laitière exprimée en valeur absolue de la perte de lait quotidienne (g/j) et la Production Laitière Totale (PLT) toutes races confondues (en italique le nombre de données par classe de PLT.

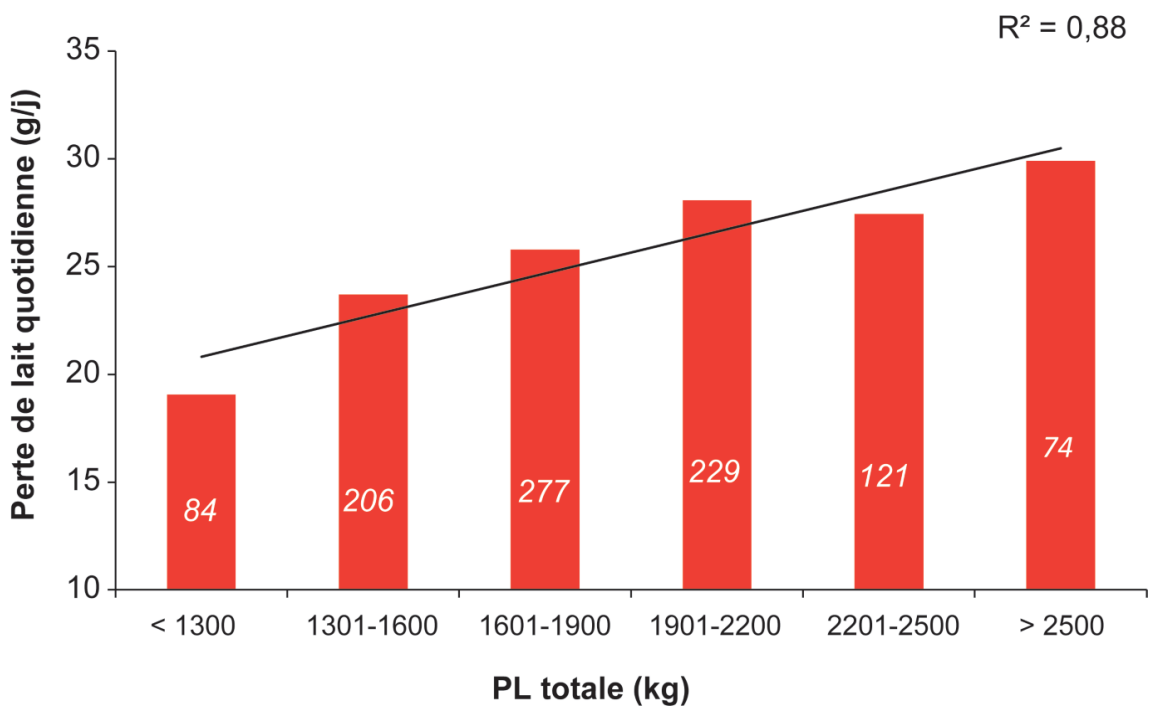

$12,3 \mathrm{~g} / \mathrm{j})$ et des charolaises $(-27,5 \pm$ $12,2 \mathrm{~g} / \mathrm{j})$ de notre base de données diffèrent significativement.

Dans la littérature, il existe une grande variabilité dans la mesure de la persistance (tableau 3 ). Sur des vaches de race hereford la diminution serait en moyenne de - 23,8 g/j (Gleddie et al 1968, Gaskins et Anderson 1980) alors que Miller et al (2006) l'ont quantifié à - $21 \pm 10 \mathrm{~g} / \mathrm{j}$ pour les primipares et à $-24 \pm 14 \mathrm{~g} / \mathrm{j}$ pour les multipares $(p>0,05)$. Ces ordres de grandeur rejoignent ainsi nos mesures. La parité est également citée comme effet majeur (Boujenane 2010). Pour cet auteur les cellules sécrétrices des vaches primipares se renouvellent mieux. Les effets de la génétique sont aussi majeurs : Jenkins et al (2000) ont montré l'influence de la race du père alors que Petit et al (1994) observent que de jeunes femelles rustiques allaitantes, grâce à leur développement corporel plus précoce, maintiennent mieux leur PL que les races «plus typées viande ». La conduite alimentaire modifie enfin la persistance : un déficit énergétique ou nutritionnel la diminue, tout comme un stress (chaleur par exemple) (Boujenane 2010).

Les primipares de notre étude ont une meilleure persistance $(-21,2 \pm 10,2 \mathrm{~g} / \mathrm{j})$ que les multipares $(-24,3 \pm 13,7 \mathrm{~g} / \mathrm{j})$ $\mathrm{p}<0,05)$. Elles se trouvent au moment du pic à un stade de lactation plus avancé.

On peut donc rechercher un effet de la période de vêlage, en faisant l'hypothèse d'une meilleure persistance des vêlages précoces qui bénéficieraient davantage des repousses d'herbe de fin d'été. Mais cet effet ne se trouve qu'à l'état de tendance peu significative, la persistance des vaches ayant vêlé précocement est de $(-23+/-11 \mathrm{~g} / \mathrm{j})$ vs $(-26+/-14 \mathrm{~g} / \mathrm{j})$ pour les vaches vêlant plus tard.

Toutes races et âges confondus, persistance et PLT sont liées (figure 7). Dans nos données et compte tenu de la sélection des individus de l'échantillon (vaches ayant une durée de lactation

Tableau 3. Importance de la baisse de production laitière en fin de lactation selon la race, la période de lactation et le niveau de production laitière au début de l'observation.

\begin{tabular}{|l|c|c|c|l|}
\hline Race & $\begin{array}{c}\text { Stade de } \\
\text { lactation (jours) }\end{array}$ & $\begin{array}{c}\text { Production laitière } \\
\text { initiale (kg/j) }\end{array}$ & $\begin{array}{c}\text { Diminution sur la } \\
\text { période (kg) }\end{array}$ & Auteurs \\
\hline Limousine & $90-180$ & 6,2 & $-1,9$ & Le Neindre et al (1976) \\
\hline Hereford x Angus & $42-154$ & 7 & $-2,2$ & Bowden (1981) \\
\hline Hereford x Friesian & $70-120$ & 11 & $-4,5$ & Baker et al (1982) \\
\hline Charolaise & $34-190$ & 8,4 & $-2,2$ & Mc Gee et al (2015) \\
\hline Angus x Friesian & $34-190$ & 12,3 & $-3,9$ & Mc Gee et al (2015) \\
\hline
\end{tabular}


longue), les vaches peu productives ont un pic et une PLT faibles mais une bonne persistance. Ainsi les vaches dont la PLT est inférieure à $1600 \mathrm{~kg}(30 \%)$ perdent en moyenne $22 \mathrm{~g}$ de lait par jour après le pic de lactation (290 lactations étudiées) et celles dont la PL est supérieure à $2200 \mathrm{~kg}(35 \%)$ en perdent $29 \mathrm{~g}$ (moyenne sur 195 lactations). L'écart maximum entre classes extrêmes (moins de $1300 \mathrm{~kg} v s$ plus de $2500 \mathrm{~kg}$ ) est ainsi de $11 \mathrm{~g} / \mathrm{j}$ de persistance.

Cette interrelation PLT/persistance semble un peu paradoxale. Un veau qui aurait une mère forte productrice devrait au fil de la lactation la solliciter davantage, car sa capacité d'ingestion de lait ne fait qu'augmenter, avec comme résultat attendu d'améliorer la persistance. Mais ce n'est pas ce qui est observé. La PLT dépend d'abord du niveau de lait au pic, et en conséquence l'effet strict de la persistance sur la croissance du veau est difficile à estimer $\left(\mathrm{r}^{2}=0,12\right)$. D'ailleurs $60 \%$ de la production totale est réalisée en première moitié de la lactation (avant le pic). Cela relativise l'impact d'une bonne persistance qui devrait être quoi qu'il arrive couplée à un bon niveau de PL pour être profitable au veau.

Ajustée à un modèle linéaire, la PLT $(\mathrm{kg})$ dépend à la fois de la PLmax $(\mathrm{kg} / \mathrm{j}$ coefficient multiplicateur 214), et de sa semaine d'occurrence par rapport au vêlage (x 19), de la persistance (x 15) et d'une valeur fixe dépendant de la race. Cette équation nécessiterait une démarche de validation pour servir en prévision.

\section{4 / Évolution de la composition du lait bu au cours de la lactation}

La composition du lait des vaches allaitantes est mal connue, car le lait obtenu par le biais de la traite n'est pas représentatif du lait bu par le veau. Celui-ci est pourtant jugé plus riche en matières grasses par Ménissier et al (1992). Peu de publications synthétisent la composition du lait mesurée dans des essais et les résultats sont parfois extrêmes avec par exemple un Taux Butyreux (TB) de $0,77 \pm 0,48 \%$ pour Contréras et al (2015), ce qui limite la crédibilité des valeurs affectées à la race utilisée. Le NRC (2016) fixe cette composition à partir de la moyenne des informations disponibles à $40 \pm 12 \mathrm{~g} / \mathrm{kg}$ pour le TB, $38 \pm 2$ pour le Taux Protéique (TP) et recommande de ne garder que les valeurs moyennes $40 \mathrm{~g}$ et $38 \mathrm{~g}$ pour toutes les races. Les recommandations de l'Inra $(1988,2007)$ proposent des valeurs légèrement supérieures pour le TB $(45 \mathrm{~g} / \mathrm{kg})$ et inférieure pour le TP $(33 \mathrm{~g} / \mathrm{kg})$.

Comme pour les vaches laitières, la composition du lait des vaches allaitantes doit évoluer au cours de la lactation et selon les conditions d'alimentation. Ainsi la saison de vêlage affecte les TP et TB avec des maximums en septembreoctobre pour le TP (plus de précipitations et de pâturage) et entre novembre et avril pour le TB (Contreras et al 2015). Les effets du stade de lactation sur les teneurs en protéines, lactose et matière grasse ont été montrés dans les travaux de Contreras et al (2015) sur des vaches charolaises et leurs croisements mais n'ont pas été observés par Cerdótes et al (2004) chez des zébus nellore.

La composition du lait serait dans une moindre mesure affectée par la race (Rodrigues et al 2014) mais ce facteur racial n'est pas retenu par le NRC (2016) Nos données n'ont pas non plus permis de montrer de différences entre les races élevées sur nos installations. En effet, la composition du lait des trois races Charolaise, Limousine et Salers semble être la même puisqu'à même variation de quantité de lait ingéré, la réponse de gain de poids du veau est identique. Par ailleurs, il est souvent souligné que compte tenu des modestes quantités de lai produites, la variation de la composition du lait bu par le veau aurait assez peu d'effets sur l'énergie qu'il ingère et sur sa croissance (Mc Gee 1997).

\section{5 / Impacts de l'alimentation de la vache}

Il est connu depuis longtemps (Jarrige 1974, Petit 1978, Agabriel et Petit 1987) que la production de lait de la vache allaitante multipare est peu sensible aux diminutions du niveau d'apports énergétiques ou globaux s'ils varient dans des limites classiques d'élevage, que ce soit en bâtiments ou au pâturage. Mais on sait aussi qu'elle peut être réduite significativement lorsqu'il s'agit de primipares, ou lorsque la durée de sous-alimentation même modérée se prolonge (Petit et Garel 1981). Ainsi des régimes alimentaires à faibles teneurs en énergie $(85 \%$ des besoins) appliqués sur 16 semaines d'essai en début de lactation, peuvent faire baisser la production de façon significative $-450 \mathrm{~g}$ par semaine $v s-50 \mathrm{~g}$ par semaine chez le témoin (vaches Hereford croisés, Bartle et al 1984). C'est pourquoi les recommandations de l'Inra (1988, 2007 et 2017 à paraitre) proposent des restrictions raisonnées des niveaux d'alimentation des vaches multipares en bon état (note au vêlage supérieure à $2,5 / 5$ et pas en deçà). C'est la réussite à la reproduction et non le niveau de lactation qui les déterminent.

Dans notre base de données, lorsque le lait bu par le veau est rapporté aux apports énergétiques disponibles pour la production de lait (apports totaux besoins d'entretien théoriques), la pente moyenne de la relation observée entre traitements intra expériences (moyennes de lots appariés vaches Charolaises) est faible soit 0,2 litre par UFL marginal ingérée (figure 8). Cependant cet effet ne doit pas être linéaire comme le suggère la figure 9 qui présente les données mesurées individuellement. Si les vaches reçoivent entre 2 et 7 UFL au-dessus de l'entretien, la production ne semble pas influencée et reste proche de 6,7 litres de lait/j (soit 3 UFL/0,45 UFL/kg de lait). En dessous de ce seuil de 2 UFL disponibles pour le lait, la sensibilité de la vache changerait. Les observations individuelles retenues dans ce sous ensemble ( $n=56$ sur 115 données) indiquent une pente interindividuelle plus forte de - 0,78 kg de lait produit par UFL ingérée en moins. L'effet serait donc presque 4 fois supérieur à l'effet moyen. Mais même dans ce sous ensemble de données, lorsque la vache ne reçoit qu'une ration couvrant l'entretien, elle produit encore 5,8 litres/j soit $71 \%$ de la valeur moyenne. On peut donc bien citer la résilience de la PL face à des écarts d'apports, comme une caractéristique particulière des vaches allaitantes multipares et cela plutôt en début de lactation. En effet, Jenkins et Ferrell (1992) ont souligné un effet " retard " du niveau d'alimentation. Dans leurs essais les temps respectifs pour atteindre la PLmax étaient de 10,9, 10,7 et 9,2 semaines pour des niveaux alimentaires de 14,3, 12,4, 10,4 UFL/j (calcul pour une vache de $700 \mathrm{~kg}$ ). Le maximum de lactation est ainsi atteint plus tardivement pour les vaches allaitantes dont les besoins sont couverts par l'alimentation. L'expression phénotypique de la PLmax est la résultante d'une interaction mère veau. (cf. \$ 3.1) étroitement liée à la capacité du veau à boire, et qui ne fait que s'accroître avec son âge. Enfin un seul essai cité par Agabriel et Petit (1987) montre chez la vache salers que la perte de lait due à une restriction alimentaire est légèrement plus accentuée en fin de lactation par rapport à celle observée lorsque la restriction intervient au début de lactation. Mais les effets qui auraient pu être attribués au veau ne sont pas connus.

\section{3 / Conséquences de la relation mère/veau sur la production laitière de la mère et la crois- sance du veau}

\section{1 / Effets de la relation mère- veau sur la production laitière}

La relation mère-veau a un rôle majeur sur la tétée et donc sur la PL de la mère. En début de lactation la capacité d'ingestion du veau est un facteur limitant, la mamelle va s'adapter pour limiter sa production, mais par la suite, c'est la capacité de production de la mère qui 
Figure 8. Effet du niveau d'énergie disponible pour la production laitière (haut vs bas) sur le lait bu (moyenne de 16 lots appariés de vaches charolaises allaitant leurs veaux (De La Torre et Agabriel 2017, ce numéro).

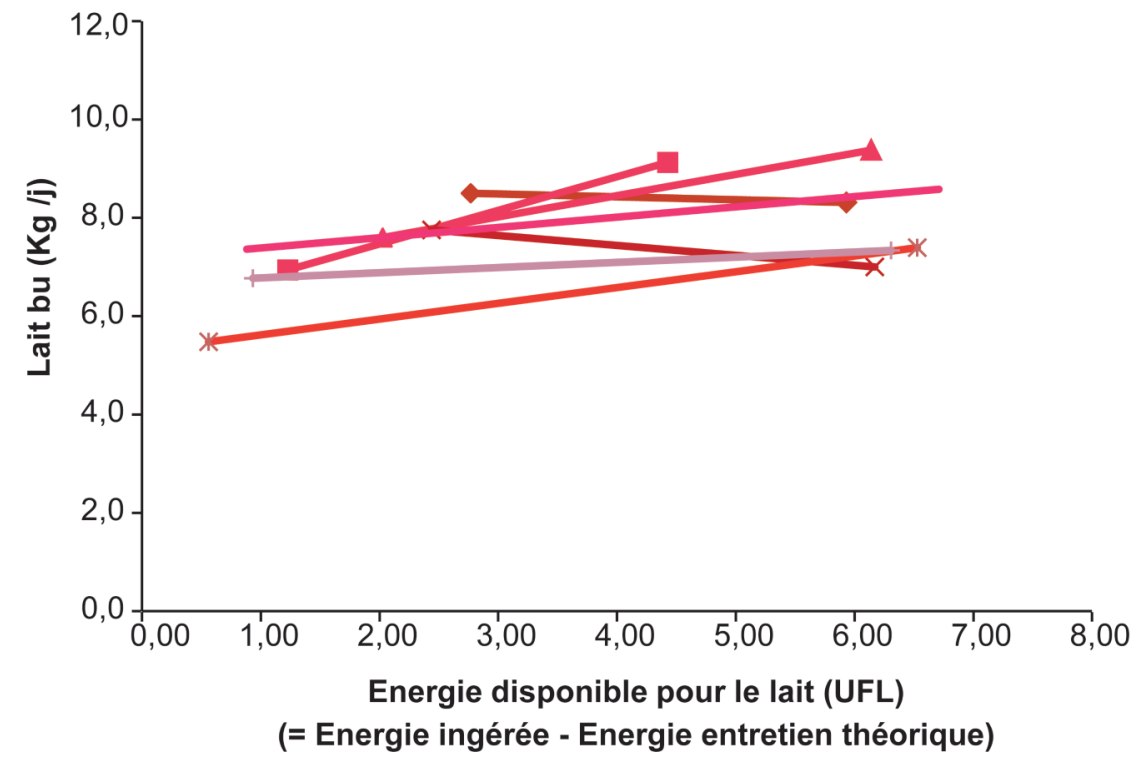

Figure 9. Relation entre l'énergie disponible pour la production laitière et la production laitière individuelle moyenne de vaches charolaises allaitant leurs veaux (De La Torre et Agabriel 2017, ce numéro).

La droite en pointillés rouge sépare les zones où les dépenses de production de lait sont couvertes ou pas par les apports d'énergie.

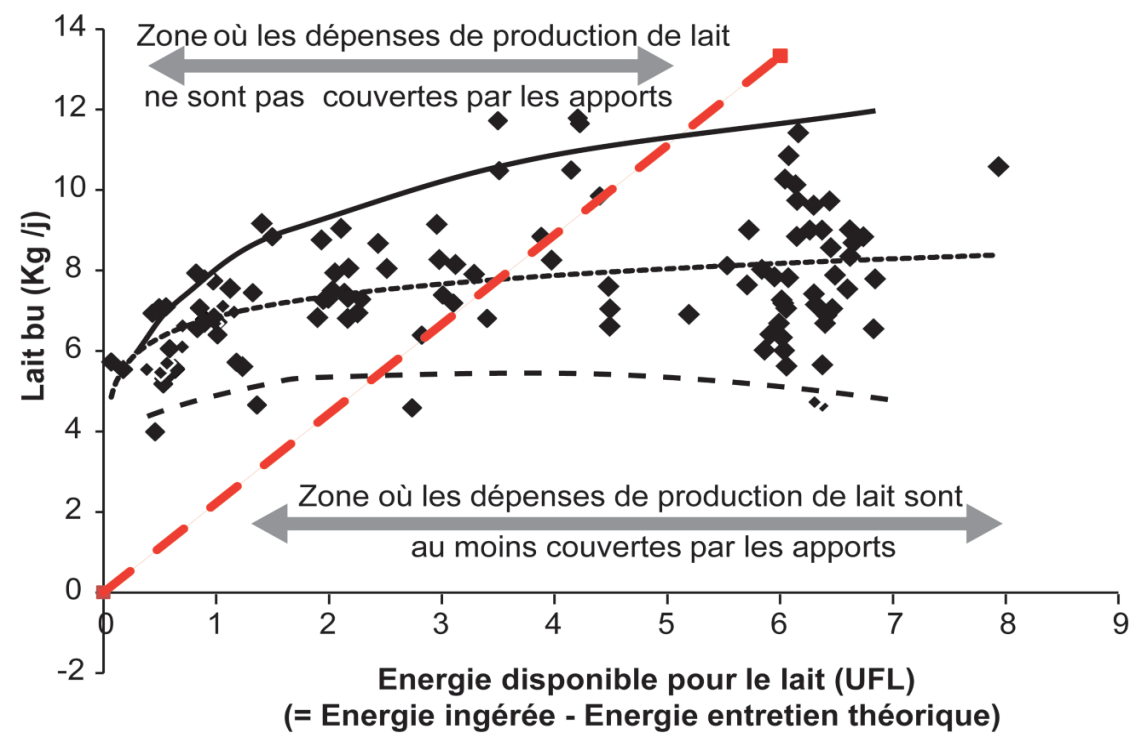

devient limitante. Cette interrelation va permettre aux vaches à fort potentiel d'augmenter leur production sous le double effet des sollicitations du veau et d'une meilleure vidange de la mamelle (Le Neindre et al 1975a). Ainsi la quantité de lait résiduel passe de $2,18 \pm$ $2,04 \mathrm{~kg}$ à $1,04 \pm 0,34 \mathrm{~kg}$ entre des vaches salers allaitant des veaux de moins de 50 jours et d'autres vaches allaitant des veaux de plus de 50 jours (Le Neindre et al 1975b). Cette relation mère-veau et l'effet du nombre de tétées sur la production bue a pu être modélisée (Blanc et al 1999). Dans les simulations du modèle, l'impact rétroactif du lait résiduel est ainsi bien mis en évidence.
Les veaux les plus lourds à la naissance ont les meilleures vitesses de croissance car leurs capacités de croissance et d'ingestion sont supérieures à celles des veaux de poids moyens (Ménissier et Petit 1984). En début de lactation, la PL dépend beaucoup de l'aptitude du veau à boire liée à son poids mais surtout de sa vitalité (Le Neindre et al 1976, Mummed 2012). La vidange de la mamelle provoquée par la tétée du veau aide au maintien de la sécrétion lactée. La stimulation du trayon engendre un relargage d'hormones (hormone de croissance, prolactine, ocytocine et adrénocorticotrophine) dans le sang qui va entretenir l'activité des acini. Lors de la tétée, en plus de la succion, les coups de tête du veau provoquent une libération d'ocytocine optimisant l'éjection du lait (Perez et al 1985, Leborgne et Tanguy 2013). Aussi, le maximum de production est d'autant plus tardif et important que l'aptitude laitière de la mère est élevée et que le veau est peu développé (Petit et al 1994) ou de faible vitalité. Avec l'avancement de la lactation, le nombre de tétées et leur durée diminuent (Le Neindre et Petit 1975a, Jammes et Djiane 1988). À un âge de quarante jours, les veaux salers élevés en stabulation réalisent en moyenne $6 \pm$ 3 tétées/j contre $3 \pm 1$ tétées par jour au pâturage à l'âge de cinq mois et demi (Le Neindre et Petit 1975a). Pour notre part (Sepchat, non publié ) nous avons observé par vidéo des veaux charolais de trois mois et leurs mères en cases de stabulation, pendant 3 années d'expérimentation. Pour un total de 45 veaux observés, le nombre de tétées par veau et par jour a varié de $2,8 \pm 0,82$ à $3,4 \pm$ 0,40 .

Enfin la PL augmente avec le nombre de veaux à allaiter (Le Neindre et al 1976, Petit et al 1978, Ménissier et al 1992). Ainsi la PL d'une vache allaitant 2 veaux augmente de $+18 \%$ (Petit et al 1978) ou de +20 à $+60 \%$ pour Ménissier et al (1992). Néanmoins, compte tenu des potentiels laitiers de nos races, la PL des mères de jumeaux ne couvre quasiment jamais les besoins de chaque veau (Ménissier et al 1992).

\section{2 / Production de lait et crois- sance du veau}

Au cours d'une lactation entière, le surplus de croissance du veau est en moyenne de $60 \mathrm{~g} /$ litre de lait bu en plus, soit un gain de poids vif de $70 \mathrm{~kg}$ pour une lactation de $2300 \mathrm{~kg}$ par rapport à une lactation de $1200 \mathrm{~kg}$, dont $31 \mathrm{~kg}$ pris entre 0 et 90 jours. La corrélation entre le GMQ et la PL cumulée diminue $\mathrm{au}$ fur et à mesure que le veau avance en âge $\left(\mathrm{R}^{2}=0,62\right.$ pour PL $0-90 \mathrm{j}$ vs 0,18 pour PL 120-210 j). La croissance du veau et la PL de la mère sont liées, mais avec une efficacité du litre de lait supplémentaire décroissante. Au-delà de $1600 \mathrm{~kg}$ de lait bu l'effet marginal du $\mathrm{kg}$ bu sur la croissance diminue (figure 10). On peut interpréter cette baisse d'efficience par l'évolution de la composition du gain de poids du veau. À ce niveau de lait bu, l'apport marginal d'énergie du lait serait utilisé pour déposer davantage de lipides coûteux en énergie et moins de protéines et d'eau (Geay et al 1987).

Quelle que soit la race, le GMQ du veau est toujours corrélé avec la PLmax $(\mathrm{r}=0,4$ à 0,6$)$, alors qu'il n'est corrélé avec la persistance que pour les charolaises $(r=0,28)$. Au final l'ensemble 
Figure 10. Relation entre la production laitière cumulée entre 0 et 210 jours de lactation ( $P L$ 0-210 j) et le gain moyen quotidien sur la même période (GMQ 0-210 j) en race salers, limousine et charolaise.

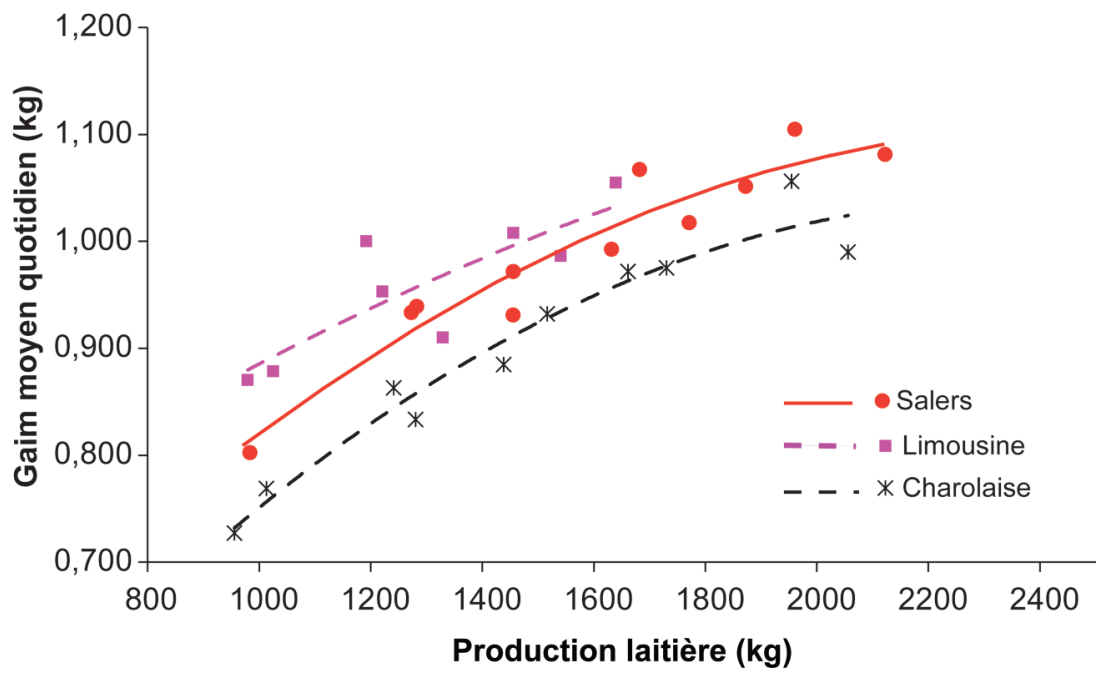

des variables constitutives de la PL présentées figure 1 peut expliquer la croissance de la naissance au sevrage. Elles s'intègrent toutes dans un modèle de régression multiple, mais n'expliquent que $28 \%$ de sa variance. Les coefficients de régression sont alors de $37 \mathrm{~g}$ de croît par kg de PL pour la PLmax et de $2,5 \mathrm{~g} / \mathrm{j}$ pour la persistance.

Au-delà des 4 premiers mois de lactation, il devient cependant difficile d'interpréter le lien entre croissance du veau et production totale, car un veau plus âgé ingère de plus en plus d'autres ingrédients (fourrages et concentrés), de 1,1 kg MS de fourrage à 100 jours à $5,3 \mathrm{~kg}$ au sevrage (Sepchat et al 2011). Au fur et à mesure du développement du rumen, l'alimentation solide complète le lait sans vraiment s'y substituer. Pour prédire à ce stade le lien entre lait bu et croissance du veau, il conviendrait d'intégrer les phénomènes de substitution entre les 3 ingrédients principaux de la ration que sont le lait, les fourrages et les concentrés. Dans une étude réalisée entre 2008 et 2011 (Garcia et al 2008, Sepchat et al 2011), trois lots (Contrôle, Concentré, Lait) de broutards salers ( $n=30)$ ont été suivis de l'âge de 3 mois jusqu'au sevrage à 9 mois. Les trois lots avaient deux tétées par jour sous leur mère et du foin à volonté. Le lot Concentré recevait en plus une complémentation en concentré et le lot Lait bénéficiait d'une tétée journalière supplémentaire sous une vache laitière. Alors que la substitution entre le foin et le lait supplémentaire reste très faible (coefficient entre 0 et 0,07 ), la substitution foin /concentré s'accroît au cours du suivi (coefficient allant de 0,23 à 0,64$)$. Il y a peu de substitution entre lait et fourrages ou concentrés, c'est toujours le lait qui est privilégié par le veau. Mais la croissance avant sevrage a été supérieure pour les lots Lait et
Concentré par rapport au lot Contrôle avec respectivement, des GMQ de 1 500, 1450 et $1160 \mathrm{~g} / \mathrm{j}$, puis identiques dans les trois lots en engraissement $(1300 \mathrm{~g} / \mathrm{j})$. Après sevrage, les animaux ont été engraissés avec un régime commun composé de $40 \%$ de foin et de $60 \%$ de concentré, puis abattus à un poids vif moyen de $650 \mathrm{~kg}$. Pour un poids de carcasse $(390 \mathrm{~kg})$ et un état d'engraissement comparables, les taurillons du lot Lait ont été abattus à un âge moyen inférieur de 10 jours à ceux du lot Contrôle. Ainsi les apports de lait et de concentré avant sevrage influencent la phase d'engraissement. L'efficacité des rations avant sevrage a été meilleure pour les lots Contrôle et Lait que pour le lot Concentré, avec un effet rémanent en début d'engraissement. Les animaux du lot Lait ont bu $800 \mathrm{~kg}$ de lait supplémentaire, mais ont ingéré $40 \%$ de concentré en moins que ceux du lot Concentré.

Cet essai illustre l'intérêt d'augmenter la quantité de lait bu, mais dans la pratique le coût du lait supplémentaire varie selon les moyens mis en œuvre pour le produire (gestion du pâturage, amélioration génétique de troupeau...). Le potentiel laitier des vaches apparaît comme un élément clé de la performance économique des itinéraires de production de broutards et taurillons. On peut s'attendre à ce que le bénéfice apporté par un apport supplémentaire en lait provenant d'une meilleure sélection des vaches sur la persistance laitière soit davantage marqué chez des races à fort potentiel de croissance (Charolaise, Limousine, Blonde d'Aquitaine...).

\section{Conclusion}

Cette étude repose sur l'analyse d'une importante base de données expérimen- tales. Elle a permis de préciser et de quantifier les variables descriptives des caractéristiques de la lactation. Elle a permis de tracer les courbes de production de référence pour les trois races Charolaise, Limousine, Salers et d'analyser l'occurrence des pics de lactation. Son originalité tient également dans l'analyse détaillée du lien entre persistance de la lactation, PL totale et croissance du veau. La persistance dans la deuxième partie de la lactation varie entre 20 et $25 \mathrm{~g}$ de perte de lait par jour. Cette valeur moyenne est affectée par la race, la parité et la place du vêlage dans la période hivernale. Les animaux au potentiel laitier faible ont une persistance supérieure en considérant un effet de la race. Cela est particulièrement vrai pour les vaches limousines qui ont le potentiel laitier le plus faible des 3 races étudiées. Comme pour les vaches traites, la persistance laitière des primipares est supérieure à celle des multipares. Son influence sur la croissance du veau est difficile à interpréter mais significative avec un rôle plus important pour les vaches au faible potentiel laitier.

L'influence de la quantité de lait bu sur la croissance du veau est désormais quantifiée et les facteurs modulant cette relation sont mis en évidence. La réponse du gain de poids du veau à un surplus de production de lait de sa mère $(60 \mathrm{~g} / \mathrm{kg}$ lait en plus) n'est pas constante au cours de la croissance. L'effet d'un kilo de lait bu supplémentaire diminue avec l'âge du veau. La composition du lait bu ne semble pas varier de façon suffisamment importante entre les races pour générer des différences de GMQ. Enfin, si la liaison entre la croissance du veau et la PL est forte et facilement quantifiable dans les 3 premiers mois de la vie du veau au-delà, il conviendra de mieux préciser la répartition entre ingestion de lait et de fourrage et leurs effets respectifs sur la croissance.

Les qualités laitières des mères sont évaluées pour l'instant au travers des performances de croissance du veau. L'index Aptitude maternelle à l'allaitement (ALait) traduit l'aptitude d'une vache à bien élever son veau de la naissance au sevrage grâce à un bon potentiel laitier et à un bon comportement maternel. Mais il serait intéressant de phénotyper plus finement et à plus large échelle l'aptitude laitière des vaches allaitantes par une évaluation plus précise de leur PL. Pour cela nous avons développé, à l'UE Herbipôle, un dispositif prototype d'estimation du lait bu par les veaux. qui combine l'identification électronique des animaux et l'automatisme de pesées des veaux. Le prototype a été mis en place en 2014 et après trois années de tests, il reste désormais à valider cette méthode, afin de disposer 
de plus d'observations dans des conditions et avec des types génétiques variés. Une fois validé, cet outil pourrait se développer dans les élevages expérimentaux et devenir une méthode de référence.

\section{Remerciements}

Aux collègues de l'Herbipôle qui ont mesuré par contrôles réguliers des veaux, la PL des vaches allaitantes. En considérant 15 minutes / tétée surveillée pour 2 personnes, la base de données ainsi constituée représente 24000 heures de travail soit 15 personnes à temps plein sur une année. Un remerciement particulier à Michel Sabatier et à Jean Paul Garel. À Christiane Espinasse pour son aide précieuse lors de l'extraction des données de la base Aladin, à Anne De La Torre pour la mise à disposition des données les plus récentes dont elle dispose, ainsi qu'à Marie Falgoux et Mathieu Auboeuf pour le travail accompli durant leur stage.

\section{Références}

Agabriel J., Petit M., 1987. Recommandations alimentaires pour les vaches allaitantes. INRA, Bull. Tech., CRZV Theix, France, 70, 153-166.

Agabriel J., De La Torre A., 2017. Ch 3.3 Beef Cows. In: INRA Feed Unit Systems for ruminants. Nozière P., Delaby L., Sauvant D. (Eds), Wageningen Press. In press.

Baker R.D., Le Du Y.L.P., Barker J.M., 1982 The influence of winter nutrition, grazing system and stocking rate on the performance of spring-calving Hereford X Friesan cows and their calves. Anim. Prod., 34, 213-224.

Bartle S.J., Preston R.L., Brown R.E., Grant R.J., 1984. Trenbolone acetate/oestradiol combinations in feedlot steers: dose response and milk production. J. Anim. Sci., 70, 1326-1332.

Belcher C., Frahm R., Belcher D., Bennett E., 1980. Comparison of machine milkout and calf nursing techniques for estimating milk yields of various two-breed cross range cows. Oklahoma Agricult. Exp. Station, 6-10.

Blanc F., Agabriel J., Sabatier P., 1999. Modélisation de la relation entre sécretion du lait et croissance du veau chez la vache allaitante. Renc. Rech. Rum., 6, 163.

Boujenane I., 2010. La courbe de lactation des vaches laitières et ses utilisations. L'Espace Vétérinaire, 92, 1-5.

Bowden D.M., 1981. Feed utilization for calf production in the first lactation by 2 years-old F1 crossbred beef cows. J. Anim. Sci., 51, 304315 .

Cerdotes L., Restle J., Alves Filho D.C., Nörnberg M.F.B., Nörnberg J.L., Heck I., Silveira M. F., 2004. Production and composition of milk of cows of four genetic groups submitted to two feeding managements during the lactation period. Rev. Brasil. Zootec., 33, 610-612.

Colleau J.J., Lefebvre J., Dupont M., Felgines C., Wimitzky M., 1983. Sélection laitière divergente en race bovine Normande. Effets sur la production de viande des taurillons. INRA., Station de Génétique quantitative et appliquée et station de Génétique factorielle. Genet. Select. Evol., $15,119-146$.

Contreras V., Bracamonte G., Bustamante L., Medina V., Rincón A., 2015. Milk composition and its relationship with weaning weight in Charolais cattle. Rev. Brasil. Zootec., 44, 207212.

Coulon J.B., Pérochon L., 2000. Évolution de la production laitière au cours de la lactation : modèle de prédiction chez la vache laitière. INRA Prod. Anim., 13, 349-360.

De La Torre A., Agabriel J., 2017. Prendre en compte l'efficience alimentaire des vaches allaitantes dans les recommandations alimentaires à travers la quantification de leurs dépenses non productives. In : Élevage bovin allaitant. Agabriel J., Renand G., Baumont R. (Eds). Dossier, INRA Prod. Anim., 30, 153-164.

D’Hour P., Petit M., Pradel P., Garel J.P., 1995. Evolution du poids et de la production laitière au pâturage de vaches allaitantes Salers et Limousines dans deux milieux. Renc. Rech. Rum., 2, 105-108.

France génétique élevage, 2011 : Les index des races bovines à viande $:$ http://fr.france-genetiqueelevage.org/Les-index-des-races-bovines-a.html

Garcia-Launay F., Garel J.P., Micol D., Agabriel J., 2008. Alimentation des broutards : ingestion et substitution entre aliments, efficacité d'utilisation de l'énergie. Renc. Rech. Rum., 15, 263-266.

Gaskins C.T., Anderson D.C., 1980. Comparison of lactation curves in Angus-Hereford, Jersey-Angus and Simmental-Angus Cows. J. Anim. Sci., 50, 828-832.

Geay Y., Micol D., Robelin J., Berge Ph., Malterre C., 1987. Recommandations alimentaires pour les bovins en croissance et à l'engrais. Bull. Tech CRZV Theix, INRA, 70,173-183.

Gleddie V.M., Berg R.T., 1968. Milk production in range beef cows and its relationship to calf gains. J. Anim. Sci., 48, 323-333.

INRA, 1988 Alimentation des Bovins, ovins et caprins. INRA Publications, Versailles, France, $471 \mathrm{p}$.

INRA, 2007. Alimentation des bovins, ovins et caprins : Besoins des animaux - valeurs des aliments. Éditions Quae, Versailles, France, 307p.

Jammes H., Djiane J., 1988. Le développement de la glande mammaire et son contrôle hormonal dans l'espèce bovine. INRA Prod. Anim., 1, 299-310.

Jarrige R., 1974. Bases physiologiques de l'alimentation des vaches allaitantes In l'exploitation des troupeaux de vaches allaitantes. In : 6ème Journée du Grenier de Theix. Numéro spécial, Bull. Tech. CRZV Theix, France, 323-343.

Jenkins T.G., Ferrell C.L., 1992. Lactation characteristics of nine breeds of cattle fed various quantities of dietary energy. J. Anim. Sci., 70, 1652-1660.
Jenkins T.G., Ferrell C.L., Roberts A.J., 2000. "Lactation and calf weight traits of mature crossbred cows fed varying daily levels of metabolizable energy ». J. Anim. Sci., 78, 7-14.

Leborgne M.C., Tanguy J.M., 2013. Reproduction des animaux d'élevage - Troisième édition. Educagri, Dijon, 465p.

Le Neindre P., 1974. Production laitière des vaches allaitantes et liaison entre cette production et la croissance de leurs veaux. In : L'exploitation des troupeaux de vaches allaitantes. Numéro spécial, Bull. Tech. CRZV Theix, INRA, France 212-230.

Le Neindre P., Dubroeucq H., 1973. Observation sur l'estimation de la production laitière des vaches allaitantes par la pesée du veau avant et après la tétée. Ann. Zootech., 22, 413422.

Le Neindre P., Petit M., 1975a. Nombre de tétées et temps de pâturage des veaux dans les troupeaux de vaches allaitantes. Ann. Zootech., 24, 553-558.

Le Neindre P., Petit M., 1975b. Note sur l'estimation de la production laitière des vaches allaitantes au début de la lactation. Ann. Zootech., 24, 559-563.

Le Neindre P., Petit M., Muller A., 1976. Production laitière des vaches allaitantes et croissance de leurs veaux (Race Limousine). Ann. Zootech., 25, 221-224.

Mc Gee M., 1997. Defining suckler systems in terms of efficiency of lean meat production and market requirements. pHD Thesis University college Dublin, Ireland, 450p.

McGee M., Drennan M.J., Caffrey P.J., 2005. Effect of suckler cow genotype on milk yield and pre-weaning calf performance. Ir. J. Agric. Food Res., 44, 185-194.

Ménissier F., Petit M., 1984. Poids et vitalité des veaux à la naissance : leurs implications zootechniques. In : 14ème Journée du Grenier de Theix. Physiologie et pathologie périnatales chez les animaux de ferme, Jarrige R. (Ed), 279-308.

Ménissier F., Sapa J., Poivey J., 1992. Les objectifs et les critères de sélection. Les qualités maternelles des ruminants allaitants : exemple des facilités de vêlage et de l'allaitement. In : Numéro Hors série, Eléments de génétique quantitative et application aux populations animales. Bibé B., Bonaïti B., Elsen J.M. Guérin G., Mallard J., Minvielle F., De Mondini L., Mulsant P., De Rochambeau H., Farce M.H. (Eds). INRA Prod. Anim., 135-145. 
Miller N., Delbecchi L., Petitclerc D., Wagner G., Talbot B., Lacasse P., 2006. Effect of stage of lactation and parity on mammary gland cell renewal. J. Dairy Sci., 89, 4669-4677.

Mondragon I., Wilton J.W., Allen O.B., Song H., 1983. Stage of lactation effects, repetabilities and influences on weaning weights of yield and composition of milk in beef cattle. Can. J. Anim. Sci., 63, 751-761.

Mummed Y., 2012. Milk yield estimation of Ogaden cattle breed based on methods of weigh-suckle-weigh and calves' growth. Trop Anim. Health Prod., 44, 785-790.

NRC, 2016. Nutrients requirements of beef cattle, $8^{\text {th }}$ Rev. Ed. Natl. Acad. Press, Washington, DC., 494p.

Perez O., Jimenez de Perez N., Poindron P., Le Neindre P., Ravault J., 1985. Relations mèrejeune et réponse prolactinique à la stimulation mammaire chez la vache : influences de la traite et de l'allaitement libre ou entravé. Rep. Nutr. Dev., 25, 605-618.

Pérochon L., Coulon J. B., Lescourret F., 1996. Modelling lactation curves of dairy cows with emphasis on individual variability. Anim. Sci., $63,189-200$

Petit M., 1978. Vaches Nourrices. In : Alimentation des ruminants, 13, 377-402. INRA Publications, Paris, France.
Petit M., 1988. Alimentation des vaches allaitantes. In : Alimentation des bovins ovins et caprins, R. Jarrige (Ed.). INRA, Paris, France, 159-184.

Petit M., Garel J.P., 1981. The effect of underfeeding in early lactation on the performances of suckled cows. Noble and MacLeod Ed, Report on suckler cow workers meeting, Dunsany, Ireland.

Petit M., Liénard G., 1988. Performance characteristics and efficiencies of various types of beef cows in French production systems. 3 . Congrès Mondial de Reproduction et Sélection des Ovins et Bovins à Viande, Paris, France.

Petit M., Garel J.P., Le Neindre P., 1978 Allaitement de deux veaux par des vaches de race Salers. II. Etude de 1'adoption. Ann. Zootech. 27, 553-569.

Petit M., Garel J.P., Agabriel J., 1984. Description de la femelle salers allaitante, Contrat d'étude INRA-Somival-UBHA OFIVAL, dans le cadre du programme d'amélioration génétique de la race Salers.

Petit M., Agabriel J., D'Hour P., Garel J.P., 1994 Quelques caractéristiques des races bovines allaitantes de type rustique. INRA Prod. Anim., 7, 235-243.

Ricordeau G., 1992. Les objectifs et les critères de sélection : Synthèse des estimations de la variabilité génétique et des liaisons entre caractères dans les différentes. In : Numéro Horssérie, Eléments de génétique quantitative et application aux populations animales. Bibé B., Bonaïti B., Elsen J.M. Guérin G., Mallard J., Minvielle F., De Mondini L., Mulsant P., De Rochambeau H., Farce M.H. (Eds). INRA Prod. Anim., 107-116.

Rodrigues P.F., Menezes L.M., Azambuja R.C., Suné R.W., Barbosa Silveira I.D., Cardoso F.F. 2014. Milk yield and composition from Angus and Angus-cross beef cows raised in southern Brazil. J. Anim. Sci., 92, 2668-2676.

Sepchat B., Lherm M., Agabriel J., Micol D., Cirié C., Egal D., Garcia-Launay F., 2011. En production de taurillons Salers, la complémentation en concentré des broutards pénalise les performances zootechniques et économiques, l'apport supplémentaire de lait les améliore. Renc. Rech. Rum., 18, 221-224.

Somerville, S., Lowman, B., 1980. A comparison of machine milking and the calf-suckling technique as methods of measuring the milk yield of beef cows. Anim. Prod., 30, 365-372.

Veysset P., Lherm M., Bebin D., 2005. Évolutions, dispersions et déterminants du revenu en élevage bovin allaitant charolais. Étude sur 15 ans (1989-2003) à partir d'un échantillon constant de 69 exploitations. INRA Prod. Anim., 18, 265-275.

\section{Résumé}

Les mesures de Production Laitière (PL) des vaches allaitantes consistent à évaluer la quantité de lait bu par le veau grâce à deux pesées répétées avant et après tétée, mais elles restent difficilement réalisables et limitées aux unités expérimentales. Une base de données expérimentales regroupant 12000 mesures de lait bu, réalisées depuis 15 ans dans les troupeaux charolais, limousins et salers de l'Herbipôle (plus de 1000 vaches contrôlées et 7 mesures au moins par vache et par lactation) a été constituée pour actualiser nos connaissances quantitatives sur la production de ces vaches et en étudier les principaux facteurs de variation. La race influence la production laitière $(1600 \pm 313 \mathrm{~kg}$ lait/lactation en race limousine, $1840 \pm 355 \mathrm{~kg}$ en race charolaise et $2250 \pm 470 \mathrm{~kg}$ en race salers) et on observe toujours environ $11 \%$ d'écart entre primipares et multipares soit environ $1 \mathrm{~kg} / \mathrm{j}$ de plus pour les multipares. Dans un système « classique » de production de broutards, avec vêlage d'hiver et conduite semi-extensive au pâturage, deux pics de production laitière sont observés. Le premier a lieu un mois après le vêlage et le second, plus important, peu après la mise à l'herbe. Ces pics traduisent une augmentation de production $+0,8$ à $+2,3 \mathrm{~kg}$ selon la race, la parité et la période de vêlage. L'analyse de la base de données a permis de quantifier la persistance de la lactation et sa variabilité (diminution de la production de lait de 17 à $27 \mathrm{~g}$ de lait bu par jour). La persistance est meilleure pour les vaches faiblement productrices $(-0,5 \mathrm{~kg}$ contre - 0,9 kg/j de lait mesuré sur un mois). Une meilleure production laitière amène un meilleur gain de poids des veaux. Au cours de la lactation entière, le surplus de gain de poids moyen quotidien des veaux est de 60 g/litre de lait bu en plus, soit $70 \mathrm{~kg}$ de gain de poids vif pour une lactation de 2300 $\mathrm{kg}$ par rapport à une lactation de $1200 \mathrm{~kg}$. Une sélection sur la production de la mère et sa persistance permettrait donc de réduire les aliments achetés pour les broutards. Cela conforte la nécessité de pouvoir mesurer la production laitière de façon plus systématique et sur toute la lactation. La production de lait de la vache allaitante est peu sensible à la diminution du niveau des apports énergétiques ou globaux si celle-ci est inférieure à 3 UFL. En effet la fonction de production laitière est priorisée quel que soit l'état d'engraissement au vêlage. Néanmoins, la production laitière peut être réduite significativement lorsqu'une durée de sous-alimentation même modérée se prolonge. Avec des vêlages d'hiver, la qualité de l'herbe ingérée au printemps couvre largement (110\%) les besoins alimentaires élevés de lactation. Ceci permet à la vache de relancer sa lactation et de reconstituer les réserves mobilisées en période hivernale et ainsi à l'éleveur d'épargner sur l'alimentation distribuée.

\section{Abstract}

\section{Milk production of suckler cows: a study of the main variation factors}

Measuring suckler cow Milk Production (MP) consists in the evaluation of the milk drunk by the calf during two repeated weighings before and after suckling. This measurement is difficult to implement and limited to experimental centers. A database was created grouping the milk drunk measured over 15 years by charolais, limousin and salers cattle from the HERBIPÔLE (more than 12,000 measurements, more than 1000 cows checked at least 7 times per cow and per lactation) in order to update our quantitative knowledge of the production of suckler cows and study the major variation factors. Breed is a major factor of production: $1600 \pm 313 \mathrm{~kg}$ milk / lactation in limousin, $1840 \pm 355 \mathrm{~kg}$ in Charolais and $2250 \pm 470 \mathrm{~kg}$ in Salers and a difference of approximately $11 \%$ can be observed 
between primiparous and multiparous - i.e. $1 \mathrm{~kg} / \mathrm{d}$. In a "classic" system of "broutard" production with calvings in the winter and semi-extensive pastures, two production peaks are observed. The first peak occurs one month after calving and the second larger peak just after access to pasture. This peak varies from 0.8 to $2.3 \mathrm{~kg}$ depending on the breed, parturition and calving period. A focus was made on the factors affecting the peak around access to pasture. The study enabled the quantification of the persistence of lactation and its variability (- 17 to $-27 \mathrm{~g}$ milk drunk/d). Persistence is best for low-producing cows (- 0.5 against $-0.9 \mathrm{~kg}$ less milk $/ \mathrm{d}$ over one month). Better MP leads to a better weight gain of the calves. Throughout lactation, the average weight gain is $60 \mathrm{~g} /$ liter of extra milk drunk, i.e. a gain in live weight of $70 \mathrm{~kg}$ for a lactation of $2300 \mathrm{~kg}$ compared to a lactation of $1200 \mathrm{~kg}$. Selection of production of the mother and lactation persistence therefore reduces food purchased for weanlings. This supports the need to measure the MP more systematically and throughout lactation. The milk production of a cow suckling her calf is not very sensitive to reductions in energetic or global supplements if the reduction is less than $3 \mathrm{UFL}$, in fact this function takes priority regardless of the state of weight gain during calving. Nevertheless, the MP can be reduced significantly in the event of a longer period of even moderate under-nourishment. In winter calvings, the quality of grass ingested in the spring largely covers $(110 \%)$ the high nutritional requirements of lactation. This allows the cow to re-establish lactation and to reconstitute the reserves mobilized during the winter period, and thus the breeder can economize on the feed distributed.

SEPCHAT B., D’HOUR P., AGABRIEL J., 2017. Production laitière des vaches allaitantes : caractérisation et étude des principaux facteurs de variation. In : Élevage bovin allaitant. Agabriel J., Renand G., Baumont R. (Eds). Dossier, INRA Prod. Anim., 30, 139-152. 
